\title{
Fundamentals and applications of the Landau-Lifshitz-Bloch equation
}

\author{
U Atxitia ${ }^{1}$, D Hinzke ${ }^{2}$ and U Nowak ${ }^{2}$ \\ 1 Fachbereich Physik and Zukunftskolleg, Universität Konstanz, D-78457 Konstanz, Germany \\ 2 Fachbereich Physik, Universität Konstanz, D-78457 Konstanz, Germany \\ E-mail: ulrich.nowak@uni-konstanz.de
}

\begin{abstract}
The influence of thermal excitations on magnetic materials is a topic of increasing relevance in the theory of magnetism. The Landau-Lifshitz-Bloch equation describes magnetisation dynamics at finite temperatures. It can be considered as an extension of already established micromagnetic methods with a comparable numerical effort. This review is a brief summary of this new field of research, with a focus on the fundamentals of the Landau-Lifshitz-Bloch equation, its connection with the stochastic Landau-Lifshitz equation, and its applications in modern magnetism.
\end{abstract}

Keywords: Landau-Lifshitz-Bloch equation, ultrafast spin dynamics, spin caloritronics

\section{Introduction}

\subsection{Motivation and brief introduction to this review}

Contemporary research into applications of magnetic materials drives this field into areas where thermal excitations are increasingly important. On the one hand this is due to the increasing success of nanotechnology, where nanostructures are especially susceptible to thermal excitations. On the other hand new methods are investigated to control these magnetic nanostructures on ever shorter time scales, with spin-polarised currents [1, 2], laser supported [3], or even all optically [4-7]. In all these new writing schemes thermal excitation plays an important role, either as a byproduct or even triggering magnetisation switching, which is the case for thermally induced magnetisation switching [8, 9]. In the new field of spin caloritronics the idea is even to exploit thermally induced magnonic spin currents in devices with new functionalities, combining spin and thermal transport properties [10].

From the theory point of view one can understand magnetic material properties based on different approaches, starting from first principles for the quantitative calculations for a given material up to the macroscopic level of domain formation. However, the detailed calculation of dynamic properties is bound to an equation of motion. Here, the most common starting point is either the Landau-Lifshitz [11] or the Gilbert equation [12], which can be shown to be mathematically equivalent. To include the effects of thermal excitation either one has to include a noise term [13] — following the idea of Langevin dynamics - or one needs to expand the equation of motion to take care of the effect of temperature on a mesoscopic level. This leads to the so-called LandauLifshitz-Bloch equation.

This brief review is about the Landau-Lifshitz-Bloch (LLB) equation, an equation with increasing relevance in modern magnetism because of its capability to describe nonequilibrium phenomena where thermal excitation is important. Analytical solutions are possible in certain limits, though the non-linear nature of the equation calls for numerical treatments. In section 2 the fundamentals of the LLB equation are introduced: the assumptions underlying their derivation as well as the connection to classical micromagnetism. Section 3 is on a multi-scale modelling approach, linking a variety of length scales in magnetism, and with this different approaches, starting from spin-density function theory and going via atomistic spin models to mesoscopic length scales where the LLB 
equation comes into play. Section 4 is on applications of the LLB equation and section 5 is a summary with outlook.

\subsection{Limits of micromagnetism at finite temperature}

The term micromagnetism refers to a continuum theory to describe magnetic phenomena. It goes back to efforts by Landau and Lifshitz as well as Brown [13] to bridge the gap between Maxwell's equations and the quantum mechanical treatment of exchange interactions as first described by Heisenberg [14]. The Gibbs free energy is formulated as a functional integral of the spatial magnetisation distribution, where the magnitude of the magnetisation is assumed constant. The energy functional then contains a continuum version of the Heisenberg exchange interaction, crystalline anisotropy energies which rest on the symmetry of the underlying lattice as well as the strength of the spin-orbit coupling of the material, a Zeeman term, and stray field contributions which follow from Maxwell's equations.

Brown then derived his famous equations [13], which follow from energy minimisation, and formulated the equilibrium conditions. With these equations domain configurations can be calculated, once again assuming constant magnitude of the magnetisation vector field. This approach is hence valid mostly for constant and lower temperatures, where longitudinal variations of the magnetisation are irrelevant, as well as any time dependence or spatial variation of the material parameters. Furthermore, the continuum approach also sets some limit regarding the size of the magnetic textures, which has to be clearly larger than the atomic structure. To take into account magnetisation dynamics an equation of motion was formulated: the Landau-Lifshitz (LL) equation.

\subsection{The Landau-Lifshitz equation}

Landau and Lifshitz [11] were the first to formulate an equation of motion for the magnetisation vector $\mathbf{m}(\mathbf{r})$. It reads

$$
\frac{1}{\gamma} \frac{\mathrm{d} \mathbf{m}}{\mathrm{d} t}=-\left[\mathbf{m} \times \mathbf{H}_{\mathrm{eff}}\right]-\alpha\left[\mathbf{m} \times\left[\mathbf{m} \times \mathbf{H}_{\mathrm{eff}}\right]\right] .
$$

The first term is a precession with the gyromagnetic ratio $\gamma=1.76 \times 10^{11}(\mathrm{Ts})^{-1}$, which can be derived from Heisenberg equation of motion in the classical limit $[15,16]$. The second part includes the relaxation of the magnetisation phenomenologically with the dimensionless damping constant $\alpha$. The damping term allows for a dissipation of energy and angular momentum. From a microscopic point of view, this dissipation can be seen as an energy and angular momentum transfer from the spin system into the electronic system and the phononic degrees of freedom [17-19].

The effective fields $\mathbf{H}_{\mathrm{eff}}(\mathbf{r})$ follow from the Gibbs free energy and can contain contributions from exchange interactions, crystalline anisotropies, and the external magnetic field, as well as the stray field. The exchange energy usually contains the isotropic exchange after Heisenberg but may also contain Dzyaloshinskii-Moriya interactions, two-site anisotropies, or a biquadratic exchange [20, 21].
An alternative damping term was suggested by Gilbert [12] but it was shown later on [13] that these two equations are mathematically identical with only small variations of the definition of $\gamma$ and $\alpha$. In the following (since this was used in the derivations of the LLB equation) we refer to the LL equation and note that with a minor redefinition of $\gamma$ and $\alpha$ all equations can be transferred to the often used LandauLifshitz-Gilbert equation.

Solving the LL equation for a given sample with realistic material properties is an important tool in magnetism [22]. Many experimental facts can be understood knowing the domain configurations in a sample in equilibrium as well as when cycling a hysteresis curve. With increasing computational power numerical solutions can often easily be found and compare well with experiments. Open source software exists and is well established in the community (e.g. OOMMF [23]). However, solving the LL equation in the static limit leads - in the best case- to ground state domain configurations, if not to metastable states in which the system might be trapped, depending on the initial conditions. Thermal properties remain an open problem.

To include the effects of a finite temperature, thermal fluctuations are sometimes added to the equation above in the spirit of Langevin dynamics [13, 24-26]. Since the dissipation term is already present one just has to add a white noise term to the effective fields, the strength of which then depends on the temperature and the damping constant. However, this approach is solely a low temperature approximation of the true thermal behaviour. This is due to the fact that a realistic spin wave dispersion depends on the lattice structure of the underlying material while the continuum theory allows only for an approximation for low wave numbers. Furthermore, varying the temperature the magnitude of the magnetisation itself is not fixed but temperature dependent, as are all material parameters.

This changes when the LL equation with Langevin dynamics is applied on an atomistic spin model, where the spins represent atomic magnetic moments arranged on a realistic lattice $[27,28]$. Now the approach agrees with spin wave theory in the classical limit with proper equilibrium properties and the known critical behaviour at the Curie temperature. Though the atomic spin is still assumed to be of constant length longitudinal fluctuations of the thermally averaged magnetisation result from averaging over the spin fluctuations. This approach is very successful but-numerically-bound to rather small sample sizes because of the atomic resolution. However, it builds the basis for the derivation of the LLB equation in section 2.1 and is an important part of the multi-scale modelling approach described in section 3.1.

\section{Fundamentals}

\subsection{The Landau-Lifshitz-Bloch equation for ferromagnets}

The Landau-Lifshitz equation (equation (1)) is the basic model that captures the main features commonly observed in magnetisation dynamics. Whereas the precession term follows from quantum mechanical considerations the dissipation term 
is purely phenomenological and defined by only one scalar parameter, $\alpha$. As a consequence the magnetisation dissipation is isotropic; it cannot account for the underlying crystal symmetries of the lattice and, as already noted in the introduction, the LL equation only describes magnetisation dynamics that conserves the magnetisation length.

To solve these drawbacks, Baryakhtar [29, 30] generalised the LL equation (equation (1)) to allow for both relaxation of the magnetisation length as well as the symmetry of the underlying lattice (see [31] for a recent review). For a simple ferromagnet, the Baryakhtar equation is given by

$$
\frac{1}{\gamma} \frac{\mathrm{d} \mathbf{m}}{\mathrm{d} t}=-\left[\mathbf{m} \times \mathbf{H}_{\mathrm{eff}}\right]-\widehat{\alpha} \mathbf{H}_{\mathrm{eff}} .
$$

Compared to the LL equation (equation (1)), the phenomenological relaxation is now defined by a tensor, $\widehat{\alpha}=\alpha_{i j}$. The effective field $\mathbf{H}_{\text {eff }}$ also contains a longitudinal term owing to the exchange interactions that ultimately allows for the relaxation of the magnetisation length.

Originally the Baryakhtar equation was conceived only for the range of temperatures below the critical temperature $T_{\mathrm{c}}$. Furthermore, the temperature dependence of $\alpha_{i j}$ was in principle unknown. The Baryakhtar equation, being phenomenological, hence lacks basic information from the microscopic spin degrees of freedom, similar to the Ginzburg-Landau theory of phase transitions [32], and indeed it was derived with similar arguments. To shed some light on this problem Garanin et al [33] theoretically investigated the dynamics of single-domain magnetic particles on the basis of analytical solutions of the Fokker-Planck equation (FPE). Later on, Garanin generalised the FPE method to derive the LLB equation for ferromagnets. The LLB equation is valid for the whole range of temperatures, and gives a correct account of the temperature dependence of the damping parameters above and below $T_{\mathrm{c}}$. A brief summary of this derivation will be the content of the next section.

2.1.1. The classical LLB equation. The derivation of the LLB equation starts from a well defined microscopic model. The dynamics of each magnetic moment of the ions in a latticethe atomistic spin-follows the stochastic LL equation. The exact solution of this many-body problem requires often numerical methods and is bound to small system size. To obtain a closed equation for the dynamics of the macroscopic magnetisation, m from such a microscopic model, Garanin made use of a couple of approximations.

First, Garanin dealt with the dynamics of a single magnetic moment $\boldsymbol{\mu}$ in an external magnetic field, $\mathbf{H}$. The underlying spin dynamics of the normalised spin vector $\mathbf{S}=\boldsymbol{\mu} / \mu_{\mathrm{s}}$ is given by

$$
\frac{1}{\gamma} \frac{\mathrm{d} \mathbf{S}}{\mathrm{d} t}=-[\mathbf{S} \times(\mathbf{H}+\boldsymbol{\zeta})]-\lambda[\mathbf{S} \times(\mathbf{S} \times \mathbf{H})] .
$$

The thermal noise is represented by the Langevin field, $\zeta$, which is characterised by white noise properties, i.e. $\left\langle\zeta_{\alpha}(t)\right\rangle=0$ and

$$
\left\langle\zeta_{\alpha}(t) \zeta_{\beta}\left(t^{\prime}\right)\right\rangle=\frac{2 \lambda k_{\mathrm{B}} T}{\mu_{\mathrm{s}} \gamma} \delta_{\alpha \beta} \delta\left(t-t^{\prime}\right)
$$

where $\alpha$ and $\beta$ are Cartesian components. Here, $k_{\mathrm{B}}$ is the Boltzmann constant and $T$ the temperature of the heat bath to which the spins are coupled, $\lambda$ is the damping parameter at the atomic level, and $\mu_{\mathrm{s}}$ the atomic magnetic moment. Note that the assumption of white noise is based on the separation of time scales: the dynamics of the magnetisation is assumed to be slower than the dynamics of the microscopic processes in the heat bath leading to the fluctuations. This assumption might be questioned on time scales below picoseconds [34].

From this rather simple microscopic model the FPE can readily be calculated. The FPE is an equation in partial derivatives in time and the spin variable $\mathbf{S}$ defined on the unitary sphere, $|\mathbf{S}|=1$, of the distribution function of $\mathbf{S}$. The solutions of the FPE give the dynamics of the distribution function, $f(\mathbf{S}, t)$. The distribution function at the stationary state, with $\partial_{t} f_{0}=0$, can be used to calculate the average value of the spin polarisation simply as $\mathbf{m}=\langle\mathbf{S}\rangle \equiv \int \mathbf{S} f_{0}(\mathbf{S}) \mathrm{d} \mathbf{S}$.

The generic solution of the FPE, $f(\mathbf{S}, t)$ can therefore be used to calculate the dynamics of $\mathbf{m}$ (see figure 1). The dynamical equation reads

$$
\frac{1}{\gamma} \dot{\mathbf{m}}=-[\mathbf{m} \times \mathbf{H}]+D \mathbf{m}-\lambda\langle\mathbf{S} \times[\mathbf{S} \times \mathbf{H}]\rangle .
$$

Here, $D$ is the diffusion coefficient of the thermal noise as given in equation (4). To obtain a closed equation of motion from equation (5) one needs to estimate the second moments of the spin variable $\left\langle S_{i} S_{j}\right\rangle$. To do so, Garanin introduced a decoupling scheme, based on the solution of the FPE, of a test distribution function, $f(\mathbf{S}, t) \sim \exp (\boldsymbol{\xi S})$, with the condition that the first moment follows equation (5), where $\boldsymbol{\xi}=\beta \mu_{\mathrm{s}} \mathbf{H}$ is the effective thermal field and $\beta=1 / k_{\mathrm{B}} T$. Still, the derived equation of motion was valid only for a paramagnetic spin in an external magnetic field $\mathbf{H}$, and yet not closed. A closed final form was derived for ferromagnets. In order to tackle the transition to ferromagnets, Garanin resorted to the meanfield approximation (MFA) to estimate the spin-spin correlations. This means that the effective field acting on each spin is assumed to be the same, $\mathbf{H}_{\mathrm{MFA}}$. In this way the solution obtained for single domain magnets was utilised by the substitution $\mathbf{H} \rightarrow \mathbf{H}_{\mathrm{MFA}}$.

In particular, the classical ferromagnetic model originally considered [35] was given by the biaxial anisotropic exchange interaction Heisenberg Hamiltonian,

$$
\mathcal{H}=-\mu_{\mathrm{s}} \mathbf{H} \sum_{i} \mathbf{S}_{i}-\frac{1}{2} \sum_{\langle i j\rangle} J_{i j} \mathbf{S}_{i} \mathbf{S}_{j}+\frac{1}{2} \sum_{\langle i j\rangle} J_{i j}\left(\eta_{x} S_{i}^{x} S_{j}^{x}+\eta_{y} S_{i}^{y} S_{j}^{y}\right)
$$

where $J_{i j}$ is the exchange interaction between spins at lattice sites $i$ and $j$. Here, $\eta_{x(y)} \ll 1$, represents the anisotropy of the exchange interactions in the $x(y)$ direction. When $\eta_{x}=\eta_{y}>0$, the preferred direction is along the $z$ axis, similar to the effect of the uniaxial anisotropy described by a term in the Hamiltonian, $\mathcal{H}_{i}=-d_{z}\left(S_{i}^{z}\right)^{2}$, where $d_{z}$ is the anisotropy constant at the atomic level. In the continuum limit, $\mathbf{H}_{\mathrm{MFA}}(\mathbf{r})$ $\left(\eta_{x}=\eta_{y}\right)$ resulting from the MFA of the Hamiltonian above reads 


$$
\mu_{\mathrm{s}} \mathbf{H}_{\mathrm{MFA}}=J_{0} \mathbf{m}+\mathbf{H}+\frac{J_{0}}{\mu_{\mathrm{s}}}\left[\frac{a_{0}^{2}}{z} \Delta \mathbf{m}-\eta\left(\mathbf{m}_{x}+\mathbf{m}_{y}\right)\right],
$$

where $a_{0}$ is the lattice constant, $z$ the number of nearest neighbours, $J_{0}=z J$, and $\Delta$ the Laplacian operator. Next, the exchange approximation is used, namely, the homogeneous exchange term, $J_{0} \mathbf{m}$, is assumed to be much larger than the other contributions. Thus at first order one can assume that $\mu_{\mathrm{s}} \mathbf{H}_{\mathrm{MFA}} \approx J_{0} \mathbf{m}$. Using the exchange approximation and after some laborious algebra a closed equation of motion saw the light and Garanin presented the final form of the LLB equation for a ferromagnet,

$$
\begin{aligned}
\frac{1}{\gamma} \frac{\mathrm{d} \mathbf{m}}{\mathrm{d} t}= & -\left[\mathbf{m} \times \mathbf{H}_{\mathrm{eff}}\right]+\alpha_{\|} \frac{\left(\mathbf{m} \cdot \mathbf{H}_{\mathrm{eff}}\right) \mathbf{m}}{m^{2}} \\
& -\alpha_{\perp} \frac{\left[\mathbf{m} \times\left[\mathbf{m} \times \mathbf{H}_{\mathrm{eff}}\right]\right]}{m^{2}} .
\end{aligned}
$$

Basically, the LLB equation depends on two damping parameters, $\alpha_{\|}$and $\alpha_{\perp}$, and the effective field, $\mathbf{H}_{\text {eff. }}$. For a ferromagnet these so-called dimensionless longitudinal and transverse damping parameters are given by

$$
\alpha_{\|}=2 \lambda \frac{T}{3 T_{\mathrm{c}}}, \quad \alpha_{\perp}=\lambda\left[1-\frac{T}{3 T_{\mathrm{c}}}\right]
$$

for $T<T_{\mathrm{c}}$, and the same with $\alpha_{\perp} \Rightarrow \alpha_{\|}$for $T>T_{\mathrm{c}}$. Here, $\lambda$ is the damping parameter that describes the coupling to the heat bath at the atomic level in equation (3). The value of the damping parameter is itself a topic of current research. Its value can be taken either from experiments or from first-principle calculations $[18,19]$.

The effective field is given by

$$
\mathbf{H}_{\mathrm{eff}}=\mathbf{H}+\mathbf{H}_{\mathrm{A}}+\mathbf{H}_{\mathrm{ex}}+ \begin{cases}\frac{1}{2 \widetilde{\chi}_{\|}}\left(1-\frac{m^{2}}{m_{\mathrm{e}}^{2}}\right) \mathbf{m}, & T \lesssim T_{\mathrm{c}} \\ -\frac{1}{\widetilde{\chi}_{\|}}\left(1+\frac{3}{5} \frac{T_{\mathrm{c}}}{T-T_{\mathrm{c}}} m^{2}\right) \mathbf{m}, & T \gtrsim T_{\mathrm{c} .}\end{cases}
$$

Here, the anisotropy field is defined as $\mathbf{H}_{\mathrm{A}}=-\left(m_{x}^{2}+m_{y}^{2}\right) / \widetilde{\chi}_{\perp}$. The longitudinal field acting along $\mathbf{m}$ is defined in turn by both the longitudinal susceptibility $\left(\widetilde{\chi}_{\|}\right)$and the zero-field equilibrium magnetisation, $m_{\mathrm{e}}$. The non-homogeneous exchange field is defined as $\mathbf{H}_{\mathrm{ex}}=A \triangle \mathbf{m}(\mathbf{r})$, where $A=J_{0} a_{0}^{2} /\left(z \mu_{\mathrm{s}}\right)$ is usually termed exchange stiffness. The link to finite temperature micromagnetism is made by considering temperaturedependent material parameters. In computer simulations, where the system is subdivided into cubic cells of lateral size $\Delta$, the micromagnetic exchange field has been shown to be [36]

$$
\mathbf{H}_{\mathrm{ex}}^{i}=-\frac{2 A(T)}{m_{\mathrm{e}}^{2} M_{\mathrm{s}}^{0} \Delta^{2}} \sum_{j \in \operatorname{neigh}(i)}\left(\mathbf{m}_{j}-\mathbf{m}_{i}\right),
$$

where $M_{\mathrm{s}}^{0}$ is the zero temperature saturation magnetisation and, importantly, $A(T)$ is the temperature dependent micromagnetic exchange stiffness.
The input parameters defining the model system, $\widetilde{\chi}_{\perp}, \widetilde{\chi}_{\|}$, $A$ and $m_{\mathrm{e}}$, are temperature dependent equilibrium properties. Their temperature dependence can be determined in a number of ways, theoretically from the MFA or from atomistic spin model simulations (see section 3), or directly from fitting to experimental data.

In the following we illustrate the method to calculate them within the MFA approach [36]. The equilibrium magnetisation is calculated via the self-consistent solution of the CurieWeiss equation, $m_{\mathrm{e}}=L\left(\beta J_{0} m_{\mathrm{e}}\right)$, where $L(x)=\operatorname{coth}(x)-1 / x$. The longitudinal susceptibility is given by

$$
\widetilde{\chi}_{\|}=\frac{\mu_{\mathrm{s}}}{J_{0}} \frac{\beta J_{0} L^{\prime}}{1-\beta J_{0} L^{\prime}}
$$

where $L^{\prime} \equiv \mathrm{d} L / \mathrm{d} x$. The transverse susceptibility can be linked to the uniaxial anisotropy constant, $K(T)$, through the relation $\widetilde{\chi}_{\perp}=M_{\mathrm{s}}(T)^{2} / 2 K(T)$. To obtain $K(T)$, at low temperature one can use the Callen-Callen scaling for single-ion anisotropy, $K(T)=K m_{\mathrm{e}}^{3}$ [37], and close to $T_{\mathrm{c}}$ the scaling $K(T)=K m_{\mathrm{e}}^{2}$ (see also [38]). The exchange stiffness $A(T)$ scales with $m_{\mathrm{e}}^{2}$ in MFA.

In the linear regime-for small deviations from equilibrium - the magnetisation dynamics can be separated into transverse and longitudinal to $\mathbf{m}$. The transverse and longitudinal dynamics are governed by the relaxation rates $\tau_{\perp}$ and $\tau_{\|}$, respectively, with

$$
\tau_{\|}=\frac{\widetilde{\chi}_{\|}\left(H_{z}, T\right)}{\gamma \alpha_{\|}}, \quad \tau_{\perp}=\frac{\widetilde{\chi}_{\perp}\left(H_{z}, T\right)}{\gamma \alpha_{\perp}} .
$$

Here, $\widetilde{\chi}_{\|}\left(H_{z}, T\right)$ and $\widetilde{\chi}_{\perp}\left(H_{z}, T\right)$ are the susceptibilities at nonzero field.

In order to validate the LLB equation, Chubykalo-Fesenko et al [39] compared the relaxation rates calculated from atomistic spin dynamics simulations and those given by equation (13). For the atomistic spin model, a system of $48^{3}$ spins in a cubic lattice with periodic boundary conditions was considered, each spin following the stochastic LL equation. To calculate the relaxation times, first thermal equilibrium was established for each temperature, in the presence of a field $\mu_{\mathrm{s}} H_{z}=0.05 \mathrm{~J}$. Then, to evaluate the transverse relaxation, all spins were simultaneously rotated by an angle of 30 degrees and the relaxation back to equilibrium, parallel to the $z$ axis, was investigated. Fitting the transverse magnetisation to an expression $m_{x}(t) \sim \cos \left(t / t_{p}\right) \exp \left(-t / \tau_{\perp}\right)$, the transverse relaxation time was calculated. The longitudinal relaxation time is usually calculated from the relaxation of the initially fully ordered spin system to thermal equilibrium. This relaxation of the magnitude of the magnetisation to equilibrium was found to be approximately exponential on longer time scales, which defined the longitudinal relaxation time $\tau_{\|}$.

Figure 2 shows the variation of the longitudinal and transverse relaxation times with temperature. The rapid increase of the longitudinal relaxation time close to $T_{\mathrm{c}}$ is known as critical slowing down [40], an effect which is characteristic of second order phase transitions. Further discussions of the role of the critical slowing down in experiments will be discussed in detail in section 4.1. The perpendicular relaxation time $\tau_{\perp}$ 


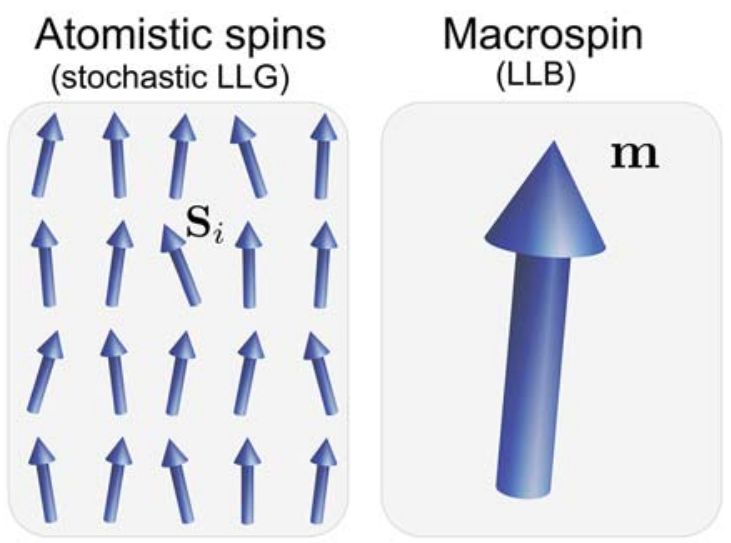

Figure 1. Left: schematic representation of the atomistic spin model. The dynamics of each atomic spin $\mathbf{S}_{i}$ is given by the stochastic Landau-Lifshitz equation of motion (equation (3)). Right: the macrospin model. The dynamics of the average magnetisation $\mathbf{m}=\sum_{i}\left\langle\mathbf{S}_{i}\right\rangle / N$ is governed by the LLB equation ( $N$, number of spins).

sharply decreases approaching the Curie temperature $T_{\mathrm{c}}$. The figure not only summarises the complex behaviour of transverse and longitudinal relaxation but also demonstrates the validity of the LLB approach in comparison to the much more complex spin dynamics simulations.

Finally, we note that the LLB equation (equation (8)) can be cast into the form proposed by Baryakhtar (equation (2)) for a damping tensor $\widehat{\alpha}(\mathbf{m})=\alpha_{0}+\mu_{i j} \hat{m}_{i} \hat{m}_{j}(\hat{\mathbf{m}}=\mathbf{m} / m)$. For $T<T_{\mathrm{c}}, \alpha_{0}=\lambda\left(1-T / 3 T_{\mathrm{c}}\right)$ and $\mu_{i j}=\lambda\left(1-T / T_{\mathrm{c}}\right)$ are the zeroand second-order relaxation tensors, i.e. the coefficients of the expansion of the tensor $\alpha_{i k}$ in powers of magnetisation. Above $T_{\mathrm{c}}$, only $\widehat{\alpha}(\mathbf{m}=0)=\alpha_{0}$ survives. Thus, the LLB equation follows the symmetry considerations proposed by Baryakhtar [31] with the advantage that, in contrast to the Baryakhtar equation, the temperature dependence of the relaxation tensors is well defined, both below and above $T_{\mathrm{c}}$.

2.1.2. The quantum LLB equation. So far we have focused our attention on the derivation and description of the classical LLB equation, for which the underlying microscopic dynamics is given by a classical spin model based on equations (3) and (4). This fact has made the classical LLB equation very popular since a direct comparison between the LLB and atomistic simulations is possible. However, classical spin models effectively assume localised magnetic moments with an infinite spin quantum number $S \rightarrow \infty$. As a consequence, at low temperatures the well known Bloch $T^{3 / 2}$ law for the magnetisation does not hold [41]. In this context, the LLB equation can incorporate the quantum nature of magnetism, as the quantum LLB (qLLB) equation was in fact derived earlier than its classical counterpart [42].

The derivation is based on the density matrix technique [43] — equivalent to the Fokker-Planck equation for classical systems-for a spin system weakly interacting with a phononic heat bath. Starting from the Schrödinger equation one can obtain a Liouville equation for the time evolution of the density operator $\hat{\rho}=|\Psi\rangle\langle\Psi|$, where $|\Psi\rangle$ is the wave function of the whole system (spin and phonons in this case). As one of

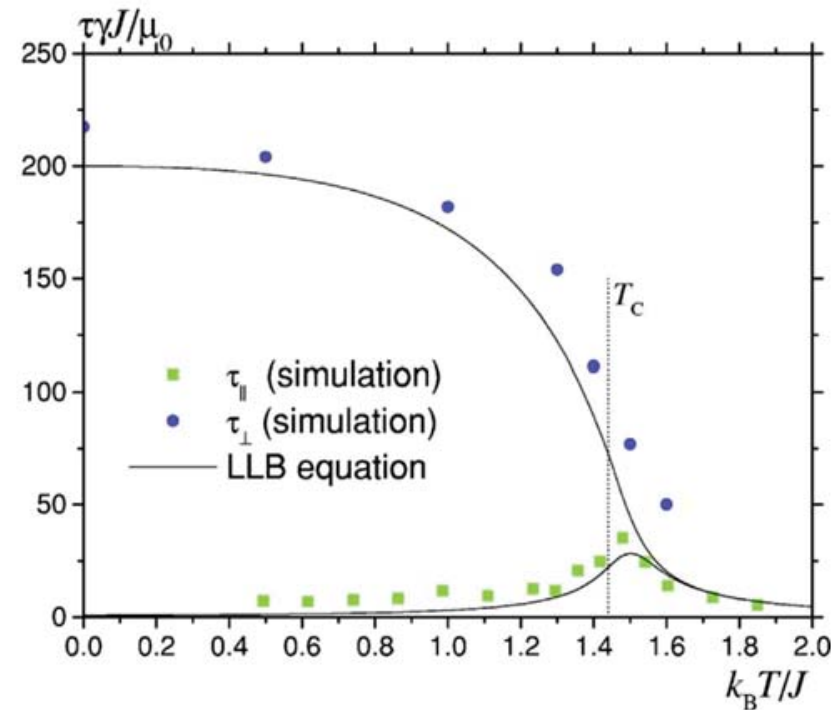

Figure 2. Temperature dependence of longitudinal and transverse relaxation times from the atomistic modelling and the LLB equation, calculated as inverse relaxation rates from the linearised LLB equation (see equations (13)). Reprinted figure with permission from [39], Copyright (2006) by the American Physical Society.

the assumptions the interaction of the spin with the heat bath is taken to be small, and neglecting any entanglement between spin and phonon allows us to factorise the density operator $\hat{\rho}$. Furthermore, it is assumed that the heat bath is in thermal equilibrium, in such a way that $\hat{\rho}(t) \cong \hat{\rho}_{\mathrm{s}}(t) \hat{\rho}_{\mathrm{b}}^{\mathrm{eq}}$ holds. After averaging over the heat bath variable one obtains the following equation of motion for the spin density operator $\hat{\rho}_{\mathrm{s}}$ [42].

$$
\begin{aligned}
\frac{\mathrm{d}}{\mathrm{d} t} \hat{\rho}_{\mathrm{s}}(t) & =-\frac{\mathrm{i}}{\hbar}\left[\hat{\mathcal{H}}_{s}, \hat{\rho}_{\mathrm{s}}(t)\right] \\
& -\frac{1}{\hbar^{2}} \int_{0}^{t} \mathrm{~d} t^{\prime} \operatorname{Tr}_{\mathrm{b}}\left[\hat{\mathcal{V}}_{\mathrm{s}-\mathrm{ph}},\left[\hat{\mathcal{V}}_{\mathrm{s}-\mathrm{ph}}\left(t^{\prime}-t\right)_{I}, \hat{\rho}_{\mathrm{s}}\left(t^{\prime}\right) \hat{\rho}_{\mathrm{b}}^{\mathrm{eq}}\right]\right]
\end{aligned}
$$

where $\operatorname{Tr}_{\mathrm{b}}$ is the trace over the bath variable, while $\hat{\mathcal{V}}_{\mathrm{s}-\mathrm{ph}}$ represents the spin-phonon interaction potential. $\hat{\rho}_{\mathrm{s}}(t)$ is written in terms of the Hubbard operators $\hat{X}^{m n}=|m\rangle\langle n|$ (where $|m\rangle$ and $|n\rangle$ are eigenvectors of $\hat{S}^{z}$, corresponding to the eigenstates $m \hbar$ and $n \hbar$, respectively), as

$$
\hat{\rho}_{\mathrm{s}}(t)=\sum_{m, n} \rho_{\mathrm{s}, m n}(t) \hat{X}^{m n},
$$

where $\rho_{\mathrm{s}, m n}(t)=\left\langle m\left|\hat{\rho}_{\mathrm{s}}(t)\right| n\right\rangle$. In particular the model Hamiltonian for a spin weakly interacting with a phononic bath reads

$$
\hat{\mathcal{H}}=\hat{\mathcal{H}}_{\mathrm{s}}+\hat{\mathcal{H}}_{\mathrm{ph}}+\hat{\mathcal{V}}_{\mathrm{s}-\mathrm{ph}}
$$

where $\hat{\mathcal{H}}_{\mathrm{s}}=-\mathbf{H} \cdot \hat{\mathbf{S}}$ with spin operator $\hat{\mathbf{S}}$ describes the spin system energy. For ferromagnets one can resort to the MFA, as for the classical LLB, with $\mathbf{H} \rightarrow \mathbf{H}^{\mathrm{MFA}}$. $\hat{\mathcal{H}}_{\mathrm{ph}}=\sum_{q} \hbar \omega_{q} \hat{a}_{q}^{\dagger} \hat{a}_{q}$ describes the phonon energy and $\hat{\mathcal{V}}_{\mathrm{s}-\mathrm{ph}}$ describes the spinphonon interaction,

$$
\hat{\mathcal{V}}_{\mathrm{s}-\mathrm{ph}}=-\sum_{q} V_{q}(\boldsymbol{\eta} \cdot \hat{\mathbf{S}})\left(\hat{a}_{q}^{\dagger}+\hat{a}_{-q}\right)-\sum_{p, q} V_{p, q}(\boldsymbol{\eta} \cdot \hat{\mathbf{S}}) \hat{a}_{p}^{\dagger} \hat{a}_{q}
$$


$\hat{a}_{q}^{\dagger}\left(\hat{a}_{q}\right)$ are the creation (annihilation) operators which create (annihilate) a phonon with frequency $\omega_{q(p)}$, where $q(p)$ stands for the wave vector $\mathbf{k}\left(\mathbf{k}^{\prime}\right)$ and the phonon polarisation. Although the spin-phonon interaction can also be taken to be anisotropic, as defined by the parameter $\boldsymbol{\eta}$, for simplicity and without loss of generality in [42] it was assumed to be isotropic, $V_{q}(\boldsymbol{\eta} \cdot \hat{\mathbf{S}})=V_{q}$ and $V_{p, q}(\boldsymbol{\eta} \cdot \hat{\mathbf{S}})=V_{p, q}$. Within this model the relaxation constants are given by

$$
\begin{gathered}
W_{1}=\sum_{q, p}\left|V_{p, q}\right|^{2} n_{p}\left(n_{q}+1\right) \pi \delta\left(\omega_{q}-\omega_{p}\right) \\
W_{2}=\sum_{q}\left|V_{q}\right|^{2}\left(n_{q}+1\right) \pi \delta\left(\omega_{q}-\omega_{0}\right) \\
+\sum_{p, q}\left|V_{p, q}\right|^{2} n_{p}\left(n_{q}+1\right) \pi \delta\left(\omega_{q}-\omega_{p}-\omega_{0}\right),
\end{gathered}
$$

where $n_{q}=\left[\exp \left(\beta \hbar \omega_{q}\right)-1\right]^{-1}$ is the Bose-Einstein distribution, and $\omega_{0}=\gamma H$. In the derivation of the qLLB further approximations were made: first, the short memory approximation, which assumes that the interaction of the spins with the phonon bath is faster than the spin interactions themselves. This means that in equation (14) the 'coarse-grained' derivative is taken over time intervals $\Delta t$ which are longer than the correlation time of the bath $\tau_{\mathrm{b}}\left(\Delta t \gg \tau_{\mathrm{b}}\right)$. Second, a secular approximation is made, where only the resonant secular terms are retained, neglecting fast oscillating terms in equation (14). A detailed discussion of the validity of these approximations can be found in the work of Nieves et al [44].

As a result of these assumptions, one arrives at a set of equations for the Hubbard operators in the Heisenberg representation, which can be connected to the spin operators $\hat{S}^{z}$, $\hat{S}^{ \pm} \equiv \hat{S}^{x} \pm \mathrm{i} \hat{S}^{y}$, and yields the following equation of motion:

$$
\begin{aligned}
\frac{\mathrm{d} \mathbf{m}}{\mathrm{dt}}= & -\gamma \mathbf{m} \times \mathbf{h} \\
& -K_{2} \frac{\tanh \left(\frac{y_{0}}{2}\right)}{\tanh \left(\frac{y}{2}\right)}\left(\frac{2(S+1) \tanh \left(\frac{y}{2}\right)}{m}-1\right) \frac{\mathbf{m} \times(\mathbf{m} \times \mathbf{h})}{m H} \\
& -2 K_{2}\left(1-\frac{\tanh \left(\frac{y_{0}}{2}\right)}{\tanh \left(\frac{y}{2}\right)} \frac{\mathbf{m} \cdot \mathbf{H}}{m H}\right) \mathbf{m} \\
& +\left(K_{2}-K_{1}\right)\left[\frac{(\mathbf{m} \times \mathbf{h})^{2}}{(m H)^{2}} \mathbf{m}+\frac{(\mathbf{m} \cdot \mathbf{H}) \mathbf{m} \times(\mathbf{m} \times \mathbf{h})}{(m H)^{2}}\right],
\end{aligned}
$$

where $y_{0}=\beta \hbar \gamma H$ and $K_{1}=W_{1}, K_{2}=\frac{1}{2}\left(1+\mathrm{e}^{-y_{0}}\right) W_{2}$.

The above form of the qLLB equation has barely been used for applications [45]. Rather, the high temperature limit, $W_{1} \approx W_{2}$, has been commonly used, which has the same form as the classical LLB in equation (8). In the qLLB, however, the damping and input parameters depend on the spin quantum number $S$. Furthermore, the intrinsic damping parameter and the microscopic relaxation constants are related by

$$
\lambda=W_{2}\left[\frac{S}{S+1}\right] \frac{\mu_{\mathrm{s}}}{k_{\mathrm{B}} T \gamma},
$$

which highlights the microscopic understanding behind the LLB equation. Another difference of the qLLB compared to its classical counterpart is the temperature dependence of the damping parameters, which below $T_{\mathrm{c}}$ is given by

$$
\begin{aligned}
& \alpha_{\|}=\lambda \frac{2 T}{3 T_{\mathrm{c}}} \frac{2 q_{s}}{\sinh \left(2 q_{s}\right)} \\
& \alpha_{\perp}=\lambda\left[\frac{\tanh \left(q_{s}\right)}{q_{s}}-\frac{T}{3 T_{\mathrm{c}}}\right],
\end{aligned}
$$

where $q_{s}=3 T_{\mathrm{c}} m_{\mathrm{e}} /(2(S+1) T)$.

The effective field $\mathbf{H}_{\text {eff }}$ necessary to solve the qLLB equation is of the same form as in equation (10). However, in contrast to the classical LLB, here the input parameters (equilibrium magnetisation $m_{\mathrm{e}}$ and susceptibilities $\widetilde{\chi}_{\|}$and $\widetilde{\chi}_{\perp}$ ) are defined by their quantum counterparts. For instance, still working in the MFA, the equilibrium magnetisation is given by the Curie-Weiss relation $m_{\mathrm{e}}=B_{\mathrm{S}}\left(\beta J_{0} m_{\mathrm{e}}\right)$, where $B_{\mathrm{S}}$ is the Brillouin function-instead of the Langevin function. In turn, the longitudinal susceptibility entering the longitudinal term of the effective field is again similar to the classical case, $\widetilde{\chi}_{\|}=\frac{\mu_{\mathrm{s}}}{J_{0}} \frac{\beta J_{0} B_{S}^{\prime}}{1-\beta J_{0} B_{S}^{\prime}}$.

Interestingly, the quantum LLB equation is not restricted to the spin-phonon interaction but was extended recently by Nieves et al [44] to include spin-electron interactions, similar to those proposed by Koopmans et al [46] in the so-called microscopic three-temperature model (M3TM). The M3TM assumes a collection of two-level spin systems $(S=1 / 2)$ and uses a self-consistent mean-field model to evaluate the macroscopic magnetisation. In the resulting system, the separation between energy levels is determined by a dynamical exchange interaction, similar to the LLB equation, which allows the authors to correctly account for high-temperature spin fluctuations. This consideration turns out to be a fundamental ingredient for the description of ultrafast demagnetisation in ferromagnets, a topic that will be discussed later on in section 4.1. Atxitia and Chubykalo-Fesenko [47] then showed that the M3TM is similar to the LLB model.

More recently, the so-called self-consistent Bloch (SCB) equation has been suggested [48]. It uses a quantum kinetic approach with the instantaneous local equilibrium approximation within the molecular-field approximation. Nieves et al [44] have compared the LLB, M3TM and SCB models, highlighting their similarities and differences, but also showing how to map these models onto each other to obtain similar results.

Similar to the classical LLB equation, the dynamics in the linear regime are defined by both the longitudinal and transverse relaxations, as given by equations (13). Notably, the transverse dynamics described by the LLB equation can be linked to the well known LLG equation, with the macroscopic (LLG-like) temperature-dependent damping, $\alpha_{\mathrm{LLG}}=\alpha_{\perp} / m_{\mathrm{e}}$. Figure 3 (top) shows the temperature dependence of $\alpha_{\mathrm{LLG}}$ for a range of spin values $S$, from $S=1 / 2$ to $S=\infty$. The transverse relaxation parameter is larger when the classical framework is used for the same system parameters, therefore the dynamics speeds up when the spin value $S$ increases. The longitudinal 

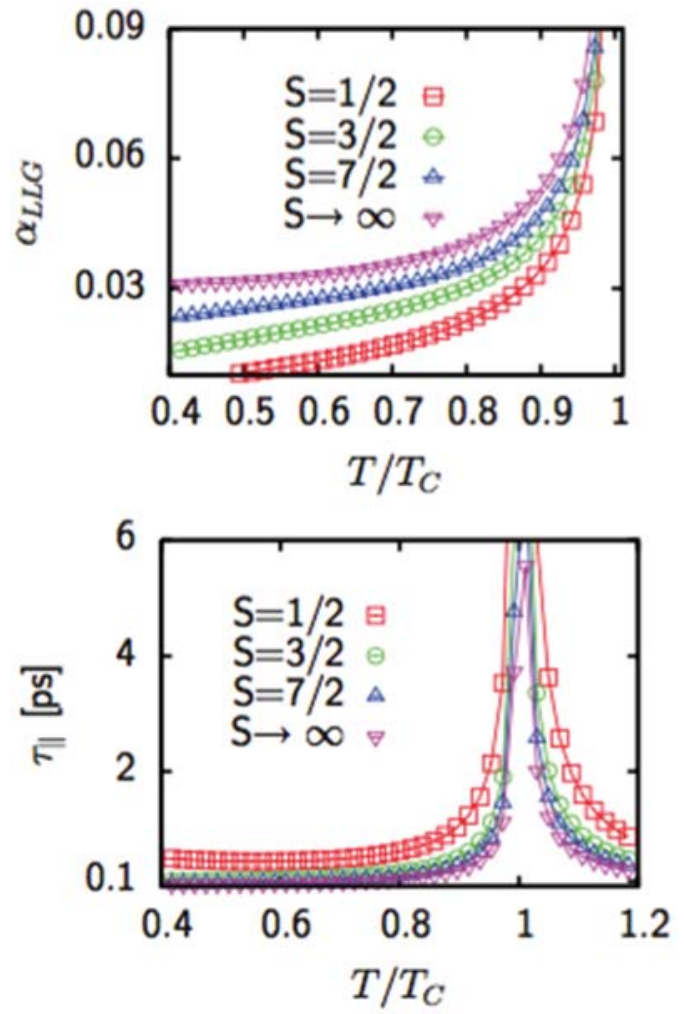

Figure 3. Spin value $S$ dependent dynamics as a function of temperature. (Top) The transverse damping parameter $\alpha_{\text {LLG. }}$ (Bottom) The longitudinal relaxation time $\tau_{\|}$. Reprinted figure with permission from [49], Copyright (2011) by the American Physical Society.

relaxation, defined by a relaxation time $\tau_{\|}$, also becomes faster with increasing spin quantum number, as shown in figure 3 (bottom). These results highlight that, although the qLLB equation is very similar in form to its classical counterpart, the qLLB dynamics depends on the quantum number $S$.

However, the advantage of the classical LLB model over the qLLB is that it allows for a parametrisation of the input parameters within a multi-scale model as will be shown in section 3 . Still it remains a true challenge to develop a full quantum multi-scale model based on the qLLB equation, where firstprinciple calculations of magnetic parameters are mapped onto a quantum Hamiltonian from which thermodynamic properties could then be calculated with quantum thermal approaches, which could finally be linked to the qLLB equation.

2.1.3. The stochastic $L L B$ equation. Both the classical and quantum versions of the LLB equation have been derived for extended systems, although at elevated temperatures the dispersion of individual trajectories of the magnetisation in ensembles of non-interacting nanoparticles plays a crucial role for the average magnetisation. In order to account for these thermal fluctuations Brown [50, 51] introduced stochastic fluctuations in the macroscopic Landau-Lifshitz-Gilbert (LLG) equation of motion. In the LLB equation, internal thermal fluctuations are already included in the temperature dependence of the input parameters. However, the effect of thermal fluctuations related to the finite volume of the particle also become important at the nanoscale.
The stochastic LLB (sLLB) equation was first introduced by Garanin and Chubykalo-Fesenko [52] based on the fluctuation-dissipation theorem. This approach worked well for temperatures not so close to $T_{\mathrm{c}}$. Later on, Evans et al introduced a slightly different version of the stochastic LLB equation [53]. The latter is given by

$$
\begin{aligned}
\frac{1}{\gamma} \frac{\mathrm{d} \mathbf{m}}{\mathrm{d} t}= & -\left[\mathbf{m} \times \mathbf{H}_{\mathrm{eff}}\right]+\frac{\alpha_{\|}}{m^{2}}\left(\mathbf{m} \cdot \mathbf{H}_{\mathrm{eff}}\right) \mathbf{m} \\
& -\frac{\alpha_{\perp}}{m^{2}}\left[\mathbf{m} \times\left[\mathbf{m} \times\left(\mathbf{H}_{\mathrm{eff}}+\boldsymbol{\xi}_{\perp}\right)\right]\right]+\boldsymbol{\xi}_{\mathrm{ad}},
\end{aligned}
$$

where $\alpha_{\|}$and $\alpha_{\perp}$ are dimensionless longitudinal and transverse damping parameters as given before in equations (9) (classical) and (22) (quantum). The effective field $\mathbf{H}_{\text {eff }}$ is again given by equation (10). Equation (23) contains two stochastic variables, $\boldsymbol{\xi}_{\perp}$, transverse to $\mathbf{m}$, which is regarded as a stochastic field added to $\mathbf{H}_{\mathrm{eff}}$, and $\boldsymbol{\xi}_{\mathrm{ad}}$, an additive isotropic torque representing magnetisation fluctuations. Evans et al [53] demonstrated that the Boltzmann distribution of $\mathbf{m}$ is only recovered by introducing the stochastic variables as in equation (23) and not by the former approach [52].

The noise in the sLLB is still considered white with first moment given by $\left\langle\xi_{\nu}^{i}(0)\right\rangle=0$ and second moments $\left\langle\xi_{\nu}^{i}(0) \xi_{\nu}^{j}(t)\right\rangle=D_{\nu} \delta_{i j} \delta(t)$, with $\nu=\mathrm{ad}, \perp$. Note that these second moments of the thermal noise variable are different to those of the stochastic LL equation, namely

$$
D_{\mathrm{ad}}=2 \frac{\alpha_{\|} k_{\mathrm{B}} T}{\gamma \mu_{\mathrm{s}}}, \quad D_{\perp}=2 \frac{\left(\alpha_{\perp}-\alpha_{\|}\right) k_{\mathrm{B}} T}{\alpha_{\perp}^{2} \gamma \mu_{\mathrm{s}}} .
$$

Interestingly, below $T_{\mathrm{c}}$ the transverse diffusion coefficient scales as $D_{\perp} \sim\left(1-T / T_{\mathrm{c}}\right)$, which implies that at temperatures close to $T_{\mathrm{c}}$ its contribution tends to zero. Above $T_{\mathrm{c}}$, where $\alpha_{\perp}=\alpha_{\|}$, it is $D_{\perp}=0$, so thermal fluctuations are solely determined by the additive noise. At low temperatures the additive thermal noise, $D_{\text {ad }} \sim 2 T / 3 T_{\text {c }}$, becomes negligible, and the stochastic LL equation is recovered. Note that with the inclusion of the noise terms the sLLB equation falls into the class of stochastic differential equations with multiplicative noise. Consequently, specialised algorithms have to be used for its numerical solution (see, e.g., $[25,54,55])$.

To illustrate the practical implication of the stochastic LLB equation, we consider switching of an FePt magnetic grain near the Curie point $T_{\mathrm{c}}$ including thermal fluctuations. We use magnetic parameters for the FePt as derived earlier [56]. The numerical calculations start with magnetic moments distributed around the equilibrium state $m_{z}=m_{\mathrm{e}} \mathbf{e}_{z}$ according to a Boltzmann distribution. Thereafter, the mean first-passage time (MFPT) is calculated, defined as the time elapsed until the magnetisation reaches the limiting value $m_{z}=-m_{\mathrm{e}} / 0.5$. The MFPT averaged over a large number of runs is the characteristic time $\tau=1 / \Gamma$, where $\Gamma$ is the magnetisation switching rate. Figure 4 shows the results obtained by the integration of the stochastic LLB (sLLB) and the stochastic LLG (sLLG) equations. The sLLG conserves magnetisation length and thus only allows for 'circular reversal', characteristic at rather low temperatures. However, at elevated temperatures the magnetisation reverses through an 'elliptical' path rather than the 


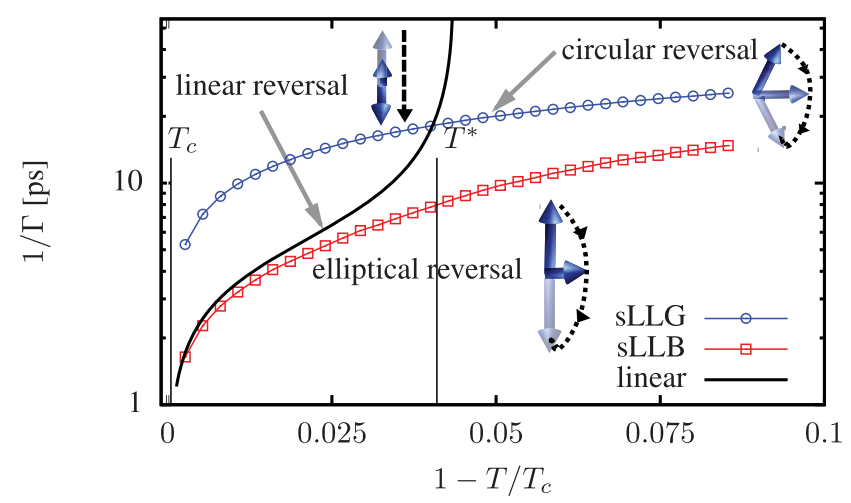

Figure 4. Reversal time as a function of temperature of a magnetic grain of $V=(5 \mathrm{~nm})^{3}$. The square symbols correspond to the solution of the sLLB equation. The solid line corresponds to the linear reversal time limit. The circles correspond to the solution of the stochastic LLG equation. The sLLB equation, in contrast to the sLLG, describes well the transition from linear reversal $\left(T^{*} \leqslant T \leqslant T_{\mathrm{c}}\right)$ to the precessional reversal $\left(T \leqslant T^{*}\right)$ regime.

circular $[52,57]$. This is due to the increasing role of the longitudinal fluctuations close to $T_{\mathrm{c}}$. At temperatures very close to $T_{\mathrm{c}}$ the transverse component of the elliptical reversal starts to disappear, leading to the so-called linear reversal. This has been shown to happen at a temperature $T^{*}$ where the transverse and longitudinal susceptibilities fulfil $\widetilde{\chi}_{\perp}\left(T^{*}\right)=2 \widetilde{\chi}_{\|}\left(T^{*}\right)$, and therefore the energy barriers associated with them are equal. For $T>T^{*}$ the reversal is more likely to go via the linear path since the energy barrier defined by $\widetilde{\chi}_{\|}$gets much smaller. This effect is enhanced in highly anisotropic magnetic nanoparticles. More insights about the linear reversal and its implications in magnetisation reversal will be given in section 4.2.

\subsection{The LLB equation for two sublattice magnets}

Pure elemental ferromagnetic materials are rare and most magnetic materials for applications are composed of more than one magnetic sublattice, partly displaying antiferromagnetic or ferrimagnetic order or building even more complex, noncollinear spin structures. Antiferromagnets and ferrimagnets are composed of at least two magnetic sublattices with their magnetic moments pointing in different directions. However, even ferromagnets can have more than one sublattice when different chemical elements are involved. Because of the increasing importance of these complex magnetic materials the LLB equation of motion for two sublattice magnets has been derived recently, and we will introduce this concept in the following.

At the microscopic level, a two lattice magnetic material is also described by the classical spin Hamiltonian in equation (6). There, all the parameters are now element specific, as schematically shown in figure 5 . The exchange interaction, $J_{i j}$, now depends on the nature of the spins at sites $i$ and $j$. If the spins are in the same sublattice $J_{i j}=J_{\nu(\kappa)}$ and between different sublattices $J_{i j}=J_{\nu \kappa}<0$ for ferrimagnets and antiferromagnets and $J_{i j}=J_{\nu \kappa}>0$ for ferromagnets. The atomic magnetic moment can also be different for each sublattice, $\mu_{\nu}$ and $\mu_{\kappa}$. The anisotropy energy will be considered as on-site
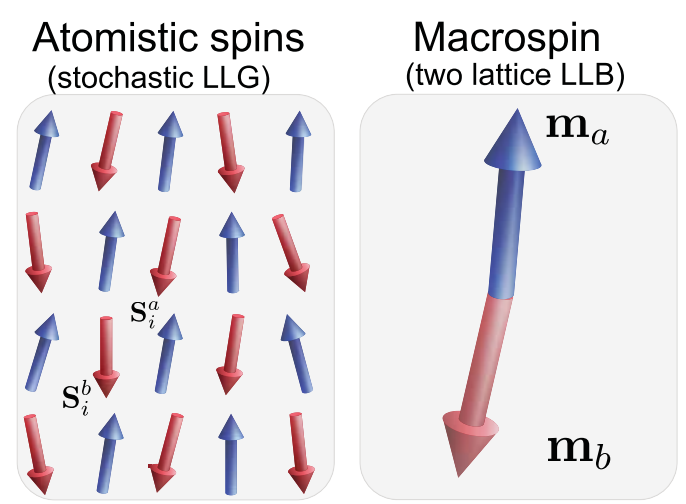

Figure 5. Left: sketch of an atomistic regular ferrimagnetic lattice. Each arrow represents a magnetic moment associated with an atomic site. Right: a macroscopic view of the averaged sublattice magnetisations $m_{\mathrm{a}}=\left\langle s_{\mathrm{a}}\right\rangle$ and $m_{\mathrm{b}}=\left\langle s_{\mathrm{b}}\right\rangle$ represented by two macrospins for each sublattice as described by the LandauLifshitz-Bloch equation.

anisotropy, and therefore it will only depend on the spin vector. The strength of the anisotropy is determined by $D_{\nu}$.

The mathematical form of the LLB equations for the two sublattice case is the same as in equation (8). However, the damping and input parameters for the two sublattice LLB equation are element specific. Below $T_{\mathrm{c}}$, the damping parameters $\alpha_{\|}^{\nu}$ and $\alpha_{\perp}^{\nu}$ are

$$
\alpha_{\|}^{\nu}=\frac{2 \lambda_{\nu}}{\beta \widetilde{J}_{0, \nu}}, \quad \alpha_{\perp}^{\nu}=\lambda_{\nu}\left(1-\frac{1}{\beta \widetilde{J}_{0, \nu}}\right)
$$

where $\widetilde{J}_{0, \nu}=J_{0, \nu}+\left|J_{0, \nu \kappa}\right| m_{e, \kappa} / m_{e, \nu}$. Here the sign of the second term does not depend on the sign of the interlattice exchange interaction, $J_{0, \nu \kappa}$. Above $T_{\mathrm{c}}$ the longitudinal and transverse damping parameters are equal and coincide with the expression [35] for the classical LLB equation of a ferromagnet above $T_{\mathrm{c}}$. In equations (25), the intrinsic damping parameters $\lambda_{\nu}$ depend on the particularities of the spin dissipation at the atomic level, and they can be the same or different for each sublattice. For example, in Py, which is composed of $\mathrm{Fe}(20 \%)$ and $\mathrm{Ni}(80 \%)$, the two elements have rather similar magnetic natures, due to a partially filled $3 d$ shell, and therefore the intrinsic damping parameters are expected to be similar. However, rare-earth-transition-metal alloys consist of two intrinsically different metals. Thus, it is a priori not clear how far their intrinsic damping parameters should be similar. Due to the inherent difficulties of the theoretical and/or experimental determination of the intrinsic damping parameters in single- or multi-element magnets this field is still a challenge for the magnetism community.

The effective field $\mathbf{H}_{\mathrm{eff}, \nu}$ for sublattice $\nu$ is defined as

$$
\begin{aligned}
\mathbf{H}_{\mathrm{eff}, \nu} & =\mathbf{H}+\mathbf{H}_{\mathrm{A}, \nu}+\frac{J_{0, \nu \kappa}}{\mu_{\nu}} \boldsymbol{\Pi}_{\kappa} \\
& +\left[\frac{1}{2 \Lambda_{\nu \nu}}\left(\frac{m_{\nu}^{2}}{m_{e, \nu}^{2}}-1\right)-\frac{1}{2 \Lambda_{\nu \kappa}}\left(\frac{\tau_{\kappa}^{2}}{\tau_{e, \kappa}^{2}}-1\right)\right] \mathbf{m}_{\nu},
\end{aligned}
$$

where $\boldsymbol{\Pi}_{\nu}=-\left[\mathbf{m}_{\kappa} \times\left[\mathbf{m}_{\kappa} \times \mathbf{m}_{\nu}\right]\right] / m_{\kappa}^{2}$ is transverse to $\mathbf{m}_{\kappa}$, and $\tau_{\nu}$ is the component of $\mathbf{m}_{\nu}$ parallel to $\mathbf{m}_{\kappa}$; in other words, 
$\boldsymbol{\tau}_{\nu}=\mathbf{m}_{\kappa}\left(\mathbf{m}_{\nu} \cdot \mathbf{m}_{\kappa}\right) / m_{\kappa}^{2}$, where $\kappa \neq \nu$. This decomposition of the fields above is sometimes neglected when investigating the magnetisation dynamics in ferrimagnets. However, when it comes to antiferromagnets, it is of paramount importance to always consider the small non-collinearities between sublattice magnetisations, as they are the source of the exchange enhanced fast dynamics characteristic of antiferromagnets [58].

The anisotropy field, $\mathbf{H}_{\mathrm{A}, \nu}$, is related to the zero-field transverse susceptibility or directly to the uniaxial anisotropy, similarly to a ferromagnet. The temperature dependence of the parameters defining the longitudinal dynamics in equation (26) is

$$
\Lambda_{\nu \nu}=\frac{1}{\widetilde{\chi}_{\|, \nu}}\left(1+\frac{J_{0, \nu \kappa}}{\mu_{0, \nu}} \widetilde{\chi}_{\|, \kappa}\right), \quad \Lambda_{\nu \kappa}=\frac{J_{0, \nu \kappa}}{\mu_{\mathrm{s}}} \frac{m_{e, \kappa}}{m_{e, \nu}} .
$$

For temperatures above $T_{\mathrm{c}}$ one can make use of the relation $\widetilde{\chi}(\epsilon)=2 \widetilde{\chi}(-\epsilon)$, where $\epsilon=1-T / T_{\mathrm{c}}$ is small. A complete expression of such terms above $T_{\mathrm{c}}$ was calculated previously by Nieves et al [59]. It is worth noting here that in the absence of coupling between sublattices, $J_{\nu \kappa}=0$, the longitudinal effective field recovers the form of a ferromagnet, $\Lambda_{\nu \nu}=1 / \widetilde{\chi}_{\|, \nu}$.

The temperature dependent parameters defining the LLB equation for two sublattices can again be calculated in the MFA. The equilibrium magnetisation of each sublattice can be obtained via the self-consistent solution of the Curie-Weiss equations $m_{\nu}=L\left(\beta\left(J_{0, \nu} m_{\nu}+\left|J_{0, \nu \kappa}\right| m_{\kappa}\right)\right)$, and the sublattice dependent longitudinal susceptibilities derived directly from them, $\chi_{\|}^{\nu}=\partial m_{\nu} / \partial H$ (for more details see [47]).

In order to validate the two-sublattice LLB equation, the transverse and longitudinal relaxation times were compared to atomistic spin model simulations. We note here that the analytical solutions of the linearised LLB equation-for small deviation from equilibrium-now give two modes of the collective dynamics; therefore, the individual element dynamics is a combination of these two modes. For the transverse dynamics, Schlickeiser et al utilised atomistic spin model simulations to perform numerical experiments to mimic ferrimagnetic resonance measurements [60]. For this, the oscillatory dynamics was decomposed into two modes, the so-called ferromagnetic mode (FMM) and the exchange mode (EXM). Analytical calculations for the frequency and effective damping of these uniform modes are usually based on two coupled macroscopic LLG equations [61, 62]. By using the two-sublattice LLB equation Schlickeiser et al [60] went beyond these earlier calculations, including thermal effects as well as avoiding further approximations. Figure 6 shows a direct comparison between the LLB model and atomistic spin model simulations for a generic ferrimagnet with a magnetic as well as an angular momentum compensation point. Similar to the experimental results [63], and unlike predictions based on the LLG equations, an increase of the effective damping at temperatures approaching the Curie temperature was found.

For the longitudinal dynamics, Atxitia et al [64] investigated the element specific longitudinal relaxation times for a $\mathrm{GdFeCo}$ ferrimagnet. Similar to the transverse modes, here the longitudinal relaxation of each sublattice is determined by a combination of two relaxation rates, $\Gamma^{+}$and $\Gamma^{-}$. Though at low temperature each rate is quite localised, $\Gamma^{+} \approx \Gamma_{\mathrm{Gd}}$ and

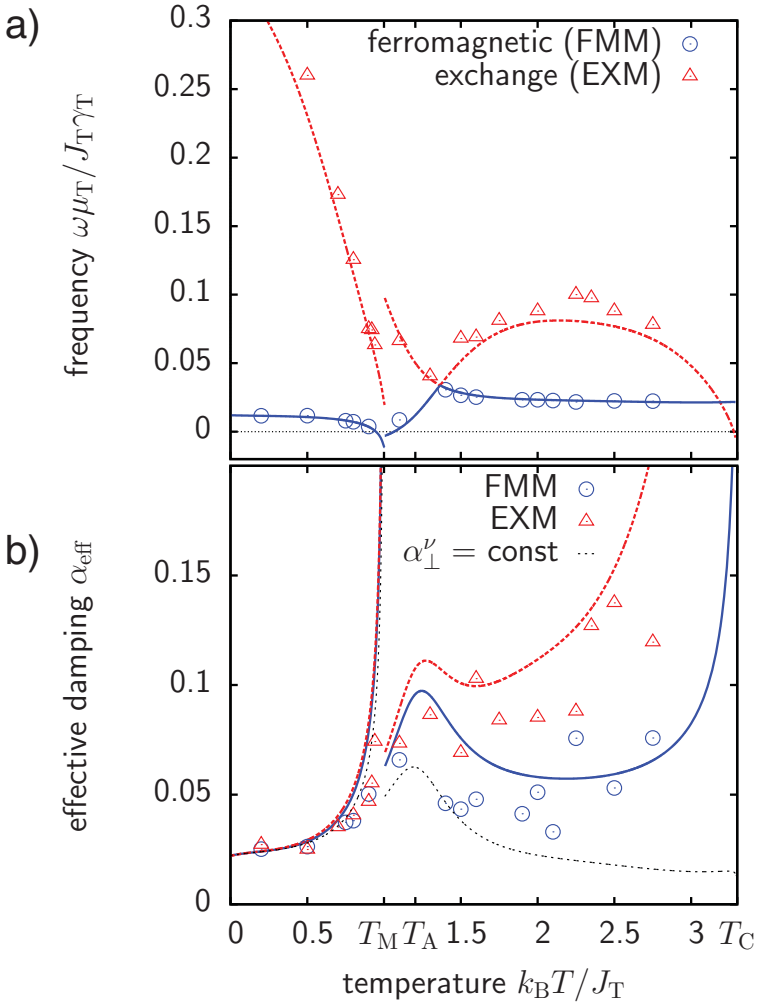

Figure 6. Temperature dependence of (a) frequencies and (b) effective damping parameters $\alpha_{\text {eff }}$ in the zero-anisotropy case. Numerically obtained data points are compared with analytical solutions. The switching of the external magnetic field $\mathbf{H}^{0}$ leads to a gap in the solutions at the magnetisation compensation point $T_{\mathrm{M}}$. Reprinted figure with permission from [60], Copyright (2012) by the American Physical Society.

$\Gamma^{-} \approx \Gamma_{\mathrm{FeCo}}$, close to $T_{\mathrm{c}}$ the interpretation is more complex. Figure 7 shows the temperature dependence of the relaxation rates as calculated from the linearised two-sublattice LLB equation. At low-to-intermediate ambient temperatures the FeCo magnetisation dynamics is faster than that of $\mathrm{Gd}$, as observed in experiments [9]. However, above a certain temperature (see yellow band), close to but below the critical temperature, the $\mathrm{Gd}$ dynamics becomes faster than that of FeCo. This behaviour has implications for the so-called transient ferromagnetic-like state and the thermally induced magnetisation switching, that we will tackle in more detail in section 4.3. These predictions were also confirmed by comparison to atomistic spin dynamics simulations [64].

\section{Multi-scale modelling for LLB dynamics}

The use of the LLB equation rests on the knowledge of certain temperature-dependent equilibrium properties, such as the spontaneous magnetisation and the susceptibilities. These can be calculated from a spin model via the MFA or by other means. However, even the spin model needs material parameters, andin more complicated cases - even the form of the Hamiltonian and the relevance of certain types of anisotropy or interaction might a priori not be clear. Often, these parameters are then treated as fitting parameters. Methods that avoid this and directly calculate material properties are called first-principle methods. 


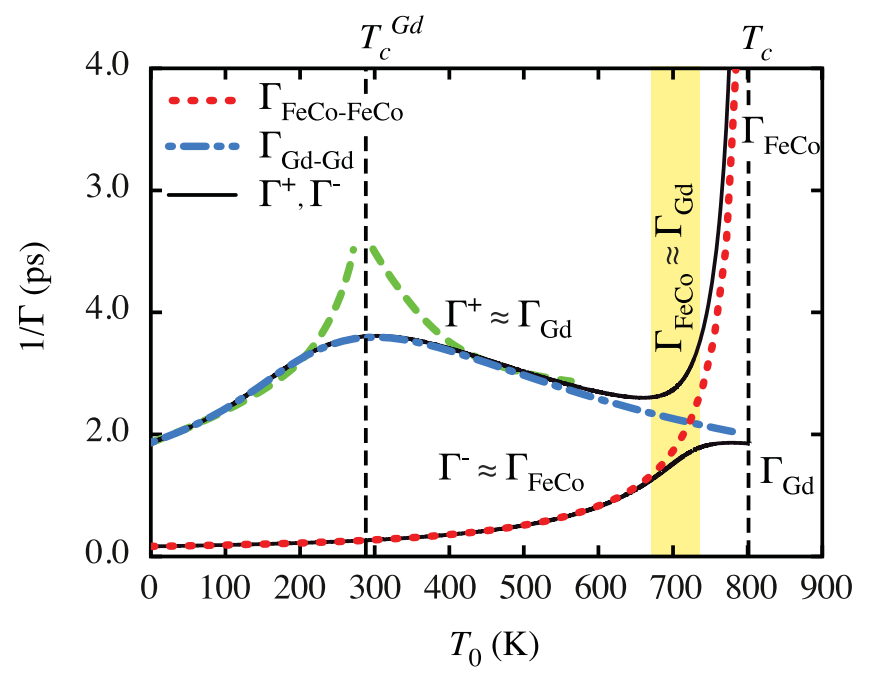

Figure 7. Longitudinal relaxation times in GdFeCo alloy as a function of temperature. At relatively low temperatures $\Gamma^{+} \approx \Gamma_{\mathrm{Gd}}$ and $\Gamma^{-} \approx \Gamma_{\mathrm{FeCo}}$. The $\mathrm{Gd}$ relaxation time presents a maximum at $T_{\mathrm{c}}^{\mathrm{Gd}}$ caused by the slowing down of the Gd fluctuations related to Gd-Gd interactions. The yellow shaded area corresponds to mixed relaxation times and both sublattices relax similarly. Close to $T_{\mathrm{c}}, \Gamma_{\mathrm{Gd}} \gg \Gamma_{\mathrm{FeCo}}$, and $\mathrm{Gd}$ sublattice magnetisation relaxes faster. Reprinted figure with permission from [64], Copyright (2014) by the American Physical Society.

The calculation of spin model parameters is mostly based on the famous approach of Liechtenstein et al [65, 66]. Different related methods have been developed in the past suitable for treating correlated systems [67, 68], relativistic effects $[20,21]$ or both of them $[69,70]$. The purpose of this section is to introduce a multi-scale modelling scheme for the LLB approach. The scheme is hierarchical in the sense that it is based on first-principle calculations to derive spin model parameters. The spin models are then, in a second step, used to calculate those equilibrium properties that are needed for the LLB equation. Finally, the LLB equation can be treated with - in the optimal case - all its parameters based on first principles, hence bridging the gaps between spin density functional theory (SDFT) and the LLB equation.

\subsection{Multi-scale modelling of ferromagnets: FePt}

The first example of a hierarchical multi-scale modelling approach using the LLB equation was the ferromagnet FePt in the layered $\mathrm{L} 1_{0}$ phase. Because of its high uniaxial anisotropy FePt is the most important ferromagnetic candidate for future data storage applications, including heat-assisted magnetic recording (for more details see section 4.2).

For the modelling of FePt, in a first step Mryasov et al constructed a microscopic spin model based on first-principle calculations of non-collinear configurations calculated by using constrained local spin density functional theory and siteresolved magneto-crystalline anisotropy (for details see [38]). In the framework of this model, it has in particular been shown that the Fe moments can be considered as localised, while the Pt induced moments have to be treated as delocalised. However, the construction of an effective classical spin Hamiltonian was finally possible considering only the Fe degrees of freedom by introduction of an additional two-ion anisotropy and modified exchange interactions between $\mathrm{Fe}$ atoms only.

The resulting Hamiltonian, with additional Zeeman energy and dipole-dipole interaction, reads

$$
\begin{aligned}
\mathcal{H}= & -\sum_{i<j}\left(J_{i j} \mathbf{S}_{i} \cdot \mathbf{S}_{j}+d_{i j}^{(2)} S_{i}^{z} S_{j}^{z}\right)-\sum_{i} d^{(0)}\left(S_{i}^{z}\right)^{2} \\
& -\sum_{i<j} \frac{\mu_{0} \mu_{\mathrm{s}}^{2}}{4 \pi} \frac{3\left(\mathbf{S}_{i} \cdot \mathbf{e}_{i j}\right)\left(\mathbf{e}_{i j} \cdot \mathbf{S}_{j}\right)-\mathbf{S}_{i} \cdot \mathbf{S}_{j}}{r_{i j}^{3}}-\sum_{i} \mu_{\mathrm{s}} \mathbf{B} \cdot \mathbf{S}_{i} .
\end{aligned}
$$

In the following this model was used in spin model simulations solving the stochastic LL equation of motion for system sizes up to about 15000 atomic spins. The isotropic exchange interactions $J_{i j}$ as well as the two-ion anisotropies $d_{i j}^{(2)}$ were taken into account for distances up to 5 unit cells. The dipoledipole interactions were calculated exactly via fast Fourier transformation (FFT) methods [71].

In order to verify the special form of the Hamiltonian and the values of the many parameters following from the SDFT calculations, the magnetic uniaxial anisotropy energy $K_{1}$ was calculated as the energy difference between simulations with the magnetisation pointing either along the easy axis or perpendicular to it. Interestingly, the temperature dependence of the magnetic anisotropy energy (MAE) was found to deviate from the expected $M(T)^{3}$ behaviour [37]. As shown in figure 8 the temperature dependences of the different contributions to the MAE coming from either the single-ion or the two-ion contribution in the Hamiltonian are different. While the first one indeed scales with $M(T)^{3}$ the latter scales with $M(T)^{2}$. Because of the different weights of these contributions, experimentally a mixed exponent, MAE $\sim M(T)^{2.1}$, was observed $[72,73]$, in agreement with the simulations. Note also that the model describes the critical temperature realistically.

Based on this effective FePt spin model Kazantseva et al introduced a hierarchical multi-scale approach bridging three methods - the first-principle calculations above, the resulting atomistic spin model and macro-spin calculations based on the LLB equation [56]. It was shown that within this multiscale approach it is possible to describe thermodynamic equilibrium and non-equilibrium magnetic properties on length scales from the single atom reaching to micrometres.

The atomistic spin simulations were performed using the FePt Hamiltonian above [38]. All the relevant equilibrium properties that have to be known for the LLB equation were calculated and parametrised: the spontaneous equilibrium magnetisation $m_{\mathrm{e}}(T)$, the exchange stiffness $A(T)$, and the susceptibilities $\tilde{\chi}_{\|}(T)$ and $\tilde{\chi}_{\perp}(T)$ (see figure 9 ). These functions are needed as input for the macrospin model in the framework of the LLB equation (8).

Note that the calculation of the thermodynamic exchange stiffness $A(T)$ for the LLB equation is less straightforward than the calculation of the magnetisation and the susceptibilities. Kazantseva et al used a result derived from the temperature dependent free energy of a domain wall and its corresponding width. For a detailed description of this calculation see [36, 74, 75]. 
a)

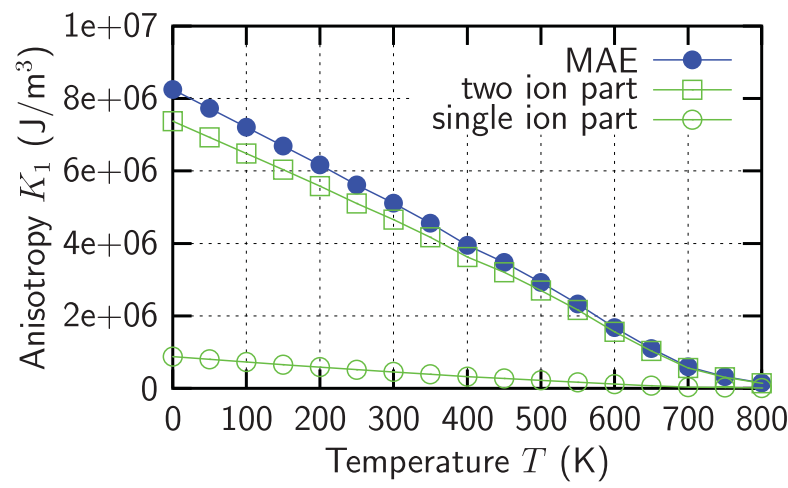

b)

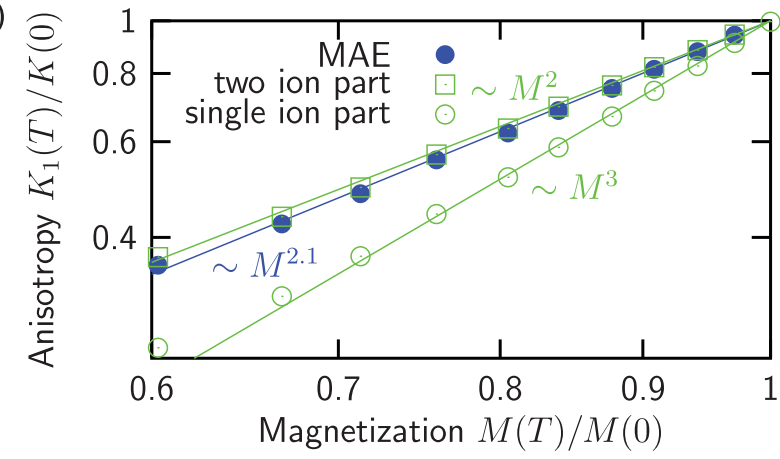

Figure 8. (a) Temperature dependence of the anisotropy $K_{1}$ from atomistic spin model simulations based on the sLLG equation of motion with the effective spin Hamiltonian in equation (28) and its single- and two-ion contributions; (b) $\log -\log$ plots for $K_{1}(T) / K_{1}(0)$ versus reduced magnetisation $M(T)$. Reproduced from [38]. (C) EDP Sciences.

Later on, Atxitia et al [76] provided detailed calculations of the temperature dependent exchange stiffness $A(T)$ via the thermally excited spin wave frequencies. To do so, two methods, numerical and analytical, were utilised. As the analytical technique the so-called classical spectral density method (CSDM) [77] was used. The CSDM allows for the calculation of the spin wave spectrum of a classical Heisenberg model as a function of temperature. As for the numerical technique, the magnetisation fluctuations around the equilibrium direction can be analysed via a Fourier analysis, in both space and time, to obtain the spin wave spectrum. The resulting spin wave spectrum is compared to the micromagnetic one, $\omega(k) \sim\left(A(T) / M_{\mathrm{s}}(T)\right) k^{2}$ where $k$ is the wavevector. In this way it was possible to extract $A(T)$. The results are presented in figure 10 as a function of the equilibrium magnetisation $m(T)$. Here, a scaling behaviour $A(m) \sim m^{\kappa}$ was found, coinciding with the results based on the numerical evaluation of the domain wall stiffness and the CSDM [77].

In general, calculating a parametrised equilibrium function by combining first principles and atomistic spin model techniques as described above is an immense numerical effort. Therefore, alternative techniques to determine the functions describing the temperature dependent input parameters to be used in the LLB equation are welcome, for instance the MFA as presented in section 2.1 [36]. Other possible techniques have not been explored so far. In section 4 results of LLB simulations based on the MFA as well as on the hierarchical multi-scale a)
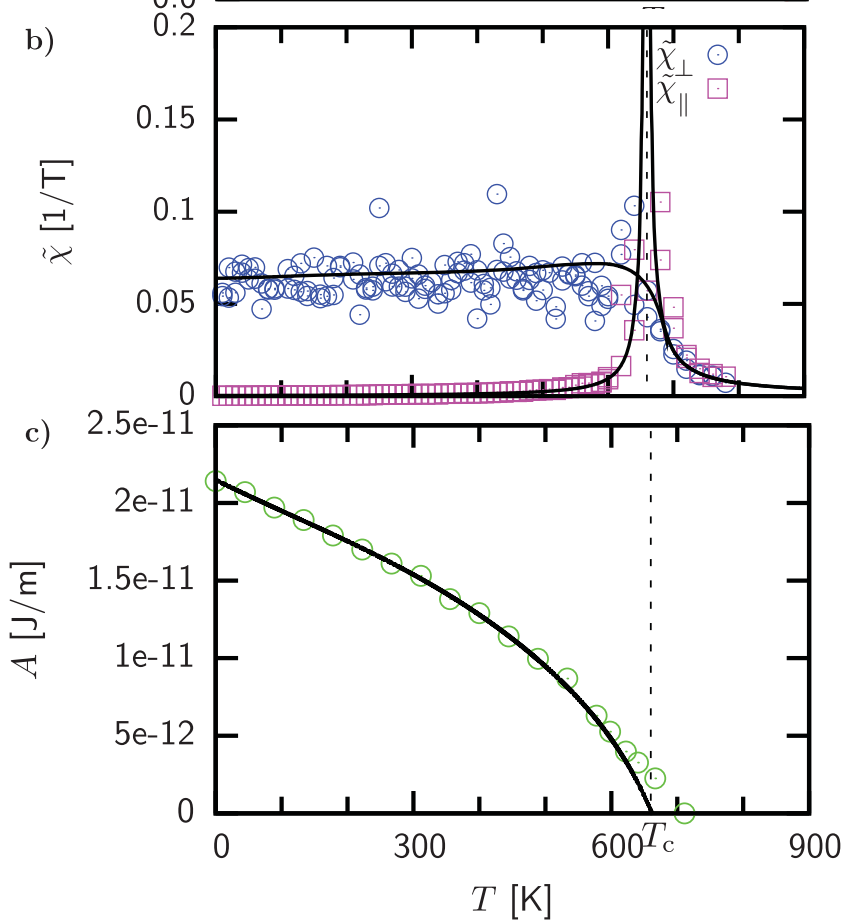

Figure 9. (a) Spontaneous equilibrium magnetisation $m_{\mathrm{e}}(T)$, (b) equilibrium parallel as well as transverse susceptibilities, and (c) exchange stiffness versus temperature for the atomistic FePt model. The solid lines represent fits to the numerical data extrapolating to $T_{\mathrm{c}}$ as for an infinite system. Reprinted figure with permission from [56], Copyright (2008) by the American Physical Society.

approach are presented. In the following section, we focus on a multi-scale approach to simulate two ferromagnetic sublattices.

\subsection{Multi-scale modelling of two sublattice ferromagnets: FeNi alloys}

In this section, we report on a hierarchical multi-scale approach to model the magnetisation dynamics of ferromagnetic random alloys composed of two different chemical constituents [78]. The developed multi-scale method was applied to FeNi (permalloy) as well as to copper-doped FeNi alloys, soft magnetic materials widely used in magnetism. Similar to FePt, firstprinciple calculations of the Heisenberg exchange integrals were linked to atomistic spin models to calculate temperaturedependent parameters, e.g. effective exchange interactions, damping parameters, and equilibrium magnetisation. The second step links the information gained from simulations of the atomistic spin model to the macroscopic two-sublattice Landau-Lifshitz-Bloch (LLB) equation [47] (section 2.2). 


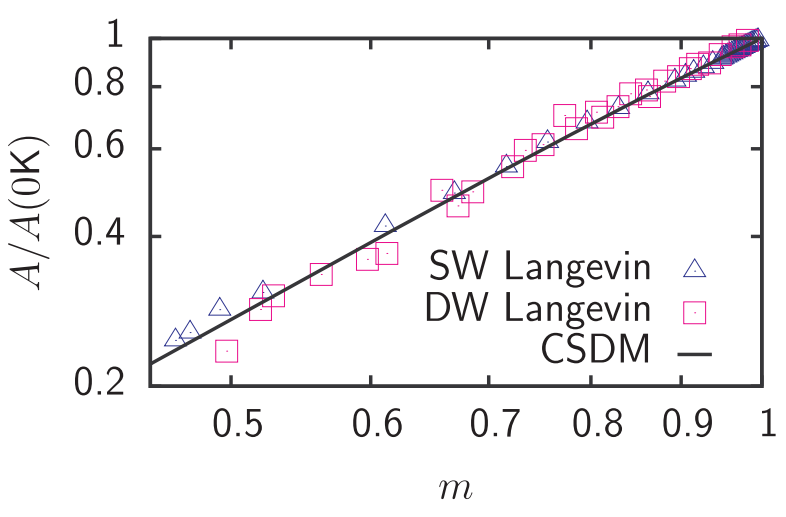

Figure 10. Scaling behaviour of the exchange stiffness as obtained from the domain wall free energy (DW Langevin). The solid line is the solution of the analytical CSDM [76]. The spin wave (SW) Langevin points are obtained from the spin wave stiffness approach based on the atomistic LLG-Langevin simulations.

To start with, an atomistic, classical spin Hamiltonian $\mathcal{H}$ was constructed on the basis of first-principle calculations to investigate the element-specific spin dynamics of FeNi alloys. In particular, three relevant alloys were studied, $\mathrm{Fe}_{50} \mathrm{Ni}_{50}, \mathrm{Fe}_{20} \mathrm{Ni}_{80}(\mathrm{Py})$ and $\mathrm{Py}_{60} \mathrm{Cu}_{40}$. This was motivated by the work of Mathias et al [79], who studied the influence of $\mathrm{Cu}$ doping on the $\mathrm{Fe}$ and $\mathrm{Ni}$ demagnetisation times in a $\mathrm{Py}_{60} \mathrm{Cu}_{40}$ alloy. To obtain the spin Hamiltonian spin-density functional theory calculations were employed to map the behaviour of the magnetic material onto an effective Heisenberg Hamiltonian. Importantly, the investigated materials are alloys. Hence, it is assumed that atoms are distributed randomly on the host fcc lattice. The effect of disorder was described by the coherent-potential approximation (CPA) [80]. The calculations of the Heisenberg exchange constants $J_{i j}$ in ferromagnets were performed by employing the magnetic force theorem [65, 66]. By using these firstprinciple methods the distance-dependent exchange constants for the FeNi alloys were calculated, i.e. the exchange between the $\mathrm{Fe}$ sublattices $(\mathrm{Fe}-\mathrm{Fe})$ and the $\mathrm{Ni}$ sublattices $(\mathrm{Ni}-\mathrm{Ni})$ as well as the $\mathrm{Fe}$ and $\mathrm{Ni}$ sublattices $(\mathrm{Fe}-\mathrm{Ni})$. The atomic magnetic moments and lattice constants for all three alloys were also calculated through the same method, for exact values [78].

Within this hierarchical multi-scale approach, the computed material parameters (the exchange constant matrix as well as the magnetic moments) were thereafter used as material parameters for numerical simulations based on the atomistic Heisenberg spin Hamiltonian, similar to equation (28). It is important to note that for the FeNi composites investigated here the alloy character was introduced as an impurity model, that is, the system is composed of classical spins $\mathbf{S}_{i}^{\epsilon}=\boldsymbol{\mu}_{i} / \mu_{s_{i}}$ with $\varepsilon$ randomly representing iron $\left(\mu_{s_{i}}=\mu_{\mathrm{Fe}}\right)$ or nickel magnetic moments $\left(\mu_{s_{i}}=\mu_{\mathrm{Ni}}\right)$ on the fcc sublattice. Importantly, for the $\mathrm{Cu}$-doped $\mathrm{Py}_{60} \mathrm{Cu}_{40}$ alloy the calculated magnetic moments on $\mathrm{Cu}$ vanish, i.e. $\mu_{\mathrm{Cu}}=0$. The atomistic spin model allowed us to calculate both thermal equilibrium and non-equilibrium properties, by numerical solutions of the stochastic LLG equation of motion. Figure 11 shows the

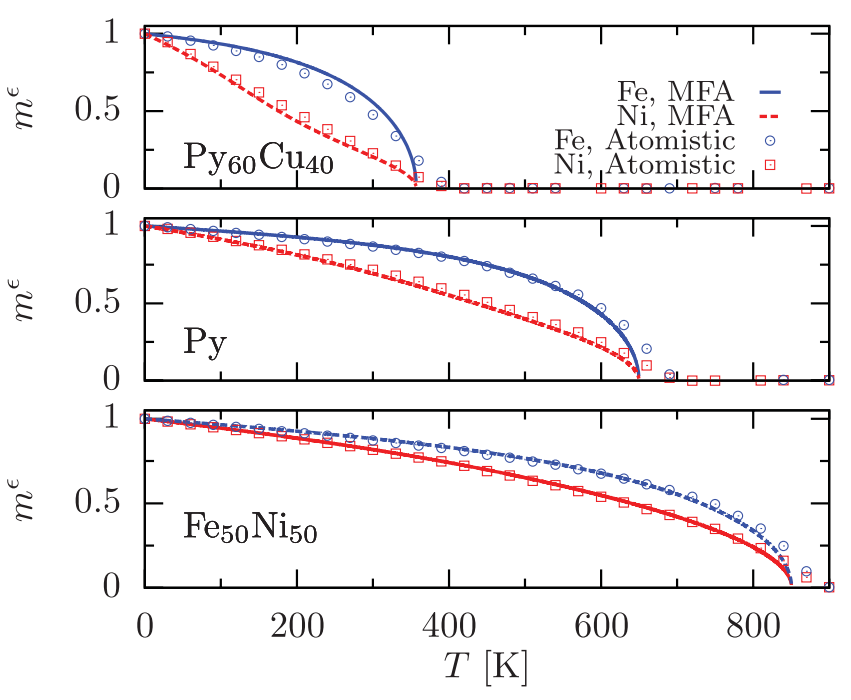

Figure 11. Element-specific zero-field equilibrium magnetisation $m^{\epsilon}$ of either Fe or Ni as a function of temperature calculated by a rescaled mean-field approximation (MFA) (lines) and by the atomistic spin dynamics simulation (open symbols). In the MFA the exchange parameters are renormalised by equalising the Curie temperatures $T_{\mathrm{c}}$ computed with atomistic simulations with those obtained from the rescaled MFA. System size $128 \times 128 \times 128$, damping parameter $\lambda=1.0$. Reprinted figure with permission from [78], Copyright (2015) by the American Physical Society.

element-specific equilibrium magnetisation $m^{\epsilon}$ of either Fe or $\mathrm{Ni}$. The calculated values of the Curie temperature compared well with known experimental values.

The link between the atomistic spin model and the LLB equation for $\mathrm{FeNi}$ alloys was made using the following set of coupled LLB equations for each reduced sublattice magnetisation $\mathbf{m}^{\epsilon}$ :

$$
\begin{aligned}
\dot{\mathbf{m}}^{\epsilon}= & -\gamma^{\epsilon}\left[\mathbf{m}^{\epsilon} \times\left\langle\mathbf{H}_{\mathrm{MFA}}^{\epsilon}\right\rangle^{\mathrm{conf}}\right]-\Gamma_{\perp}^{\epsilon} \frac{\left[\mathbf{m}^{\epsilon} \times\left[\mathbf{m}^{\epsilon} \times \mathbf{m}_{0}^{\epsilon}\right]\right]}{\left(m^{\epsilon}\right)^{2}} \\
& -\Gamma_{\|}^{\epsilon}\left(1-\frac{\mathbf{m}^{\epsilon} \mathbf{m}_{0}^{\epsilon}}{\left(m^{\epsilon}\right)^{2}}\right) \mathbf{m}^{\epsilon} .
\end{aligned}
$$

Here, $\mathbf{m}_{0}^{\epsilon}=\mathcal{L}\left(\xi_{0}^{\epsilon}\right) \boldsymbol{\xi}_{0}^{\epsilon} \xi_{0}^{\epsilon}$ is the transient (dynamical) magnetisation to which the non-equilibrium magnetisation $\mathbf{m}^{\epsilon}$ tends to relax, and $\boldsymbol{\xi}_{0}^{\epsilon} \equiv \beta \mu^{\epsilon}\left\langle\mathbf{H}_{\mathrm{MFA}}^{\epsilon}\right\rangle^{\text {conf }}$ is the thermal reduced field. This form of the LLB equation is not closed-the relaxation coefficients depend on the actual magnetisation value. However it is possible to integrate it numerically. The advantage of using equation (29) is that some approximations which lead to the final one-sublattice LLB equation are not involved and therefore the comparison to spin model simulations is more accurate. Furthermore, the link to atomistic spin models only requires the multi-scale estimation of the MFA fields, $\left\langle\mathbf{H}_{\mathrm{MFA}}^{\epsilon}\right\rangle^{\text {conf }}$. As a downside, its integration into the micromagnetic theory is hardly possible. The parallel $\left(\Gamma_{\|}^{\epsilon}\right)$ and perpendicular $\left(\Gamma_{\perp}^{\epsilon}\right)$ relaxation rates in equation (29) are given by

$$
\Gamma_{\|}^{\epsilon}=\Lambda_{\mathrm{N}}^{\epsilon} \frac{1}{\xi_{0}^{\epsilon}} \frac{\mathcal{L}\left(\xi_{0}^{\epsilon}\right)}{\mathcal{L}^{\prime}\left(\xi_{0}^{\epsilon}\right)} \quad \text { and } \quad \Gamma_{\perp}^{\epsilon}=\frac{\Lambda_{\mathrm{N}}^{\epsilon}}{2}\left(\frac{\xi_{0}^{\epsilon}}{\mathcal{L}\left(\xi_{0}^{\epsilon}\right)}-1\right)
$$


$\Lambda_{\mathrm{N}}^{\epsilon}=2 \gamma^{\epsilon} \lambda^{\epsilon} /\left(\beta \mu^{\epsilon}\right)$ is the characteristic diffusion relaxation rate. The damping parameters $\lambda^{\epsilon}$ have the same origin as those used in the atomistic simulations.

The attention of the FeNi work was placed on the dynamics of the magnetisation modulus, hence the first and the second terms on the right-hand side of equation (29) describing the transverse motion of the magnetisation can be neglected. Consequently, the LLB equation reads

$$
\dot{m}^{\epsilon}=-\Gamma_{\|}^{\epsilon}\left(m^{\epsilon}-m_{0}^{\epsilon}\right)
$$

In spite of the fact that the form of equation (31) is similar to that of the well known Bloch equation, the quantity $m_{0}=m_{0}^{\epsilon}\left(m^{\epsilon}, m^{\delta}\right)$ (with $\delta$ the second type of element) is not the equilibrium magnetisation but changes dynamically through the dependence of the effective field $\left\langle\mathbf{H}_{\mathrm{MFA}}^{\epsilon}\right\rangle^{\text {conf }}$ on both sublattice magnetisations. The mean field acting on each site $\mathbf{S}_{i}^{\epsilon}$ can be separated into two contributions: (a) the contribution from neighbours of the same type $j^{\epsilon}$ and (b) those of the other type $j^{\delta}$, and hence

$$
\mu^{\epsilon}\left\langle\mathbf{H}_{\mathrm{MFA}}^{\epsilon}\right\rangle^{\mathrm{conf}}=\sum_{\epsilon^{\epsilon}} \boldsymbol{J}_{j^{\epsilon}}^{\epsilon}\left\langle\mathbf{S}_{j^{\epsilon}}\right\rangle+\sum_{\epsilon^{\delta}} \boldsymbol{J}_{j^{\delta}}^{\epsilon}\left\langle\mathbf{S}_{j^{\delta}}\right\rangle .
$$

When the homogeneous magnetisation approximation is applied (i.e. $\left\langle\mathbf{S}_{j^{\mathrm{Fe}}}\right\rangle=\mathbf{m}^{\mathrm{Fe}}$ and $\left\langle\mathbf{S}_{j^{\mathrm{Ni}}}\right\rangle=\mathbf{m}^{\mathrm{Ni}}$ for all sites) one

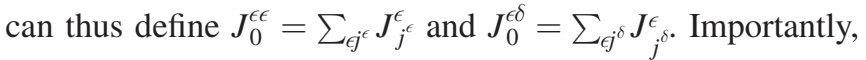
these values are those calculated via first-principle methods. Here, a further step to link the spin impurity model to the LLB macrospin approach was to map it to a regular spin lattice, where the unit cell contains the two spin species, $\mathrm{Fe}$ and $\mathrm{Ni}$, and the exchange interactions among them are weighted in terms of the concentration of each species. The equilibrium magnetisation of each sublattice $m_{\mathrm{e}}^{\epsilon}$ can be obtained via the self-consistent solution of the Curie-Weiss equations $m_{\mathrm{e}}^{\epsilon}=\mathcal{L}\left(\beta \mu^{\epsilon}\left\langle\mathbf{H}_{\mathrm{MFA}}^{\epsilon}\right\rangle^{\text {conf }}\right)$. However, a quantitative comparison between the equilibrium properties of both standard MFA and atomistic spin model calculations is usually not possible. This is due to the fact that the Curie temperature gained with the MFA approach is overestimated due to the inherent poor approximation of the spin-spin correlations. However, rescaling the exchange parameters conveniently in such a way that the Curie temperatures (calculated with the MFA approach and atomistic simulations) are identical leads to a good agreement of the two methods. Figure 11 shows good agreement of the calculated $m_{\mathrm{e}}^{\epsilon}(T)$ using the MFA and the atomistic spin model for the three system studied in the present work. The exchange interaction normalisation is $J_{0, \mathrm{MFA}}^{\epsilon \delta} \simeq(1.65 / 2) J_{0}^{\epsilon \delta}$, for $\mathrm{Fe}_{50} \mathrm{Ni}_{50}$ and Py. For $\mathrm{Py}_{60} \mathrm{Cu}_{40}$, the normalisation of the exchange parameters gives the relation $J_{0, \text { MFA }}^{\epsilon \delta}=(1.78 / 2) J_{0}^{\epsilon \delta}$.

In the following, Hinzke et al studied the reaction of the element-specific magnetisation to a sudden change of temperature (a step function) in Py as well as in Py diluted with $\mathrm{Cu}$ [78]. With the first temperature step the system was heated to $T=0.8 T_{\mathrm{c}}$ and with the second step it was cooled to $T_{\text {pulse }}=0.5 T_{\text {c }}$. This heat pulse roughly mimics the effect of heating with a ultrashort laser pulse. The first part of the

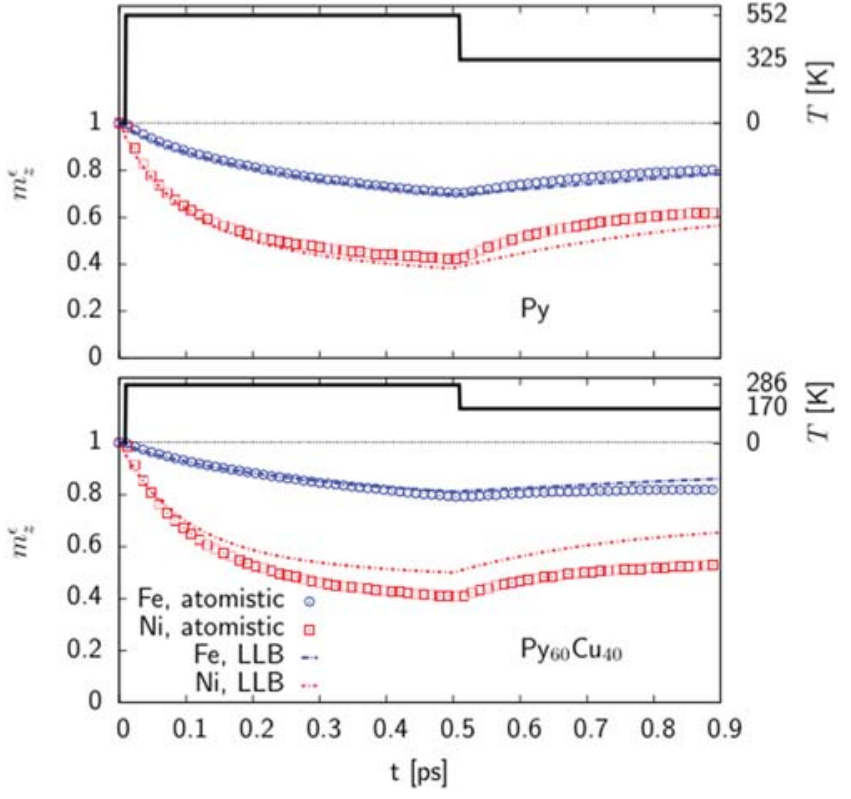

Figure 12. Calculated $z$-component of the normalised elementspecific magnetisation $m_{z}^{\epsilon}$ versus time for Py (top panel) and $\mathrm{Py}_{60} \mathrm{Cu}_{40}$ (bottom panel). In both cases the quenching of the element-specific magnetisations for $\mathrm{Fe}$ and $\mathrm{Ni}$ due to a temperature step of $T_{\text {pulse }}=0.8 T_{\mathrm{c}}$ is shown, computed with atomistic Langevin spin dynamics (open symbols) as well as LLB simulations (lines). System size $64 \times 64 \times 64$, damping parameter $\lambda=0.02$. Reprinted figure with permission from [78], Copyright (2015) by the American Physical Society.

temperature step triggers the demagnetisation while the second one triggers the remagnetisation process. Once again, an atomistic spin model based on first-principle calculations was simulated as well as a two-macro-spin LLB, to investigate the de- and remagnetisation of the two sublattices after the application of the step-like heat pulse.

The reaction of the $\mathrm{Fe}$ and $\mathrm{Ni}$ sublattice magnetisations is shown in figure 12. After the temperature is suddenly raised the two sublattices relax to their corresponding new equilibrium values of the sublattice magnetisations $m^{\epsilon}\left(T_{\text {pulse }}\right)$. Note that these equilibrium values are different for the two sublattices, in agreement with the temperature-dependent equilibrium element-specific magnetisations shown in figure 11. Furthermore, it was shown that the demagnetisation time after excitation with a temperature pulse is faster for Ni than for Fe for the first $200 \mathrm{fs}$, while for times longer than $200 \mathrm{fs}$ both elements demagnetise at the same rate. Experiments on Py suggest that the time shift between distinct and similar demagnetisation rates in Py is around 10-70 fs [79].

A lot of work has been focused recently on the question of which parameters define the demagnetisation dynamics after a laser pulse (a topic of discussion in the next section). For single-element ferromagnets, Kazantseva et al [81] estimated that the time scale for the demagnetisation processes should be limited by $\tau_{\text {demag }} \approx \mu_{\mathrm{s}} /\left(2 \lambda \gamma k_{\mathrm{B}} T_{\text {pulse }}\right)$, namely the strength of the thermal field provided by the pulse. For two sublattice magnets, assuming that the damping constants $\lambda$ and gyromagnetic ratios $\gamma$ are equal, it was hence argued that the demagnetisation time only depends on the different magnetic 
moments of the constituent materials [82]. However, within the LLB framework, Hinzke et al linked the dynamics to the equilibrium thermodynamic properties through the ratio

$$
\frac{\tau^{\mathrm{Ni}}}{\tau^{\mathrm{Fe}}}=\frac{\lambda^{\mathrm{Fe}}}{\lambda^{\mathrm{Ni}}} \frac{\mu^{\mathrm{Ni}}}{\mu^{\mathrm{Fe}}} \frac{\kappa_{\mathrm{Ni}}}{\kappa_{\mathrm{Fe}}} .
$$

Here, $\kappa$ is the coefficient defining the linear decrease of element-specific magnetisation at low temperature, $m_{\epsilon}(T)=1-\kappa_{\epsilon} T / T_{\mathrm{c}}$. This analytical relation, directly derived from the two-sublattice LLB equations, was tested against atomistic spin model simulations for the three FeNi alloys, showing an excellent agreement [78].

\section{Applications}

Since its derivation the LLB equation has attracted increasing attention because of its broad range of applications in modern magnetism. Some of these are connected to photo-induced processes in magnetic materials, where the heating effect is relevant. However, further non-equilibrium phenomena exist as well, e.g. when temperature gradients are applied, where the LLB equation is a valuable basis for the understanding of the induced dynamics. The following sections give an overview of a range of activities where the LLB equation has been applied successfully.

\subsection{Laser induced demagnetisation dynamics}

The dynamics that can be induced with ultrashort laser pulses in the few tens to hundreds of femtoseconds range has developed to become one of the most important investigative tools in solid-state physics and material science. In 1996 Beaurepaire et al demonstrated that the magnetic response to such a laser pulse is on a sub-picosecond time scale, much fast than was expected at that time [83]. This work initiated intensive research in the new field of ultrafast spin dynamics [6, 7].

Optical excitations of magnetic systems by ultrashort laser pulses lead to a non-equilibrium between the temperatures of the electron gas, $T_{\mathrm{e}}$, and of the lattice, $T_{\mathrm{ph}}$, that relaxes via electron-phonon scattering. The corresponding dynamics is usually described in terms of the so-called two-temperature model (2TM), that ignores any possible non-equilibrium behaviour within the electron and phonon systems. The minimal 2TM can be written as [84]

$$
\begin{gathered}
C_{\mathrm{e}} \frac{\mathrm{d} T_{\mathrm{e}}}{\mathrm{d} t}=G_{\mathrm{ep}}\left(T_{\mathrm{e}}-T_{\mathrm{ph}}\right)+P_{0}(t) \\
C_{\mathrm{ph}} \frac{\mathrm{d} T_{\mathrm{ph}}}{\mathrm{d} t}=-G_{\mathrm{ep}}\left(T_{\mathrm{e}}-T_{\mathrm{ph}}\right) .
\end{gathered}
$$

The 2TM assumes that part of the energy from the laser pulse, $P_{0}(t)$, is absorbed by the electron system. Due to the usually low electron heat capacity, $C_{\mathrm{e}}$, the maximum electron temperature could go up to thousands of Kelvin, whereas the phonon temperature remains low because of its rather high heat capacity, $C_{\mathrm{ph}}$. The electron-phonon coupling $\left(G_{\mathrm{ep}}\right)$ drives both systems towards an enhanced, common temperature on the

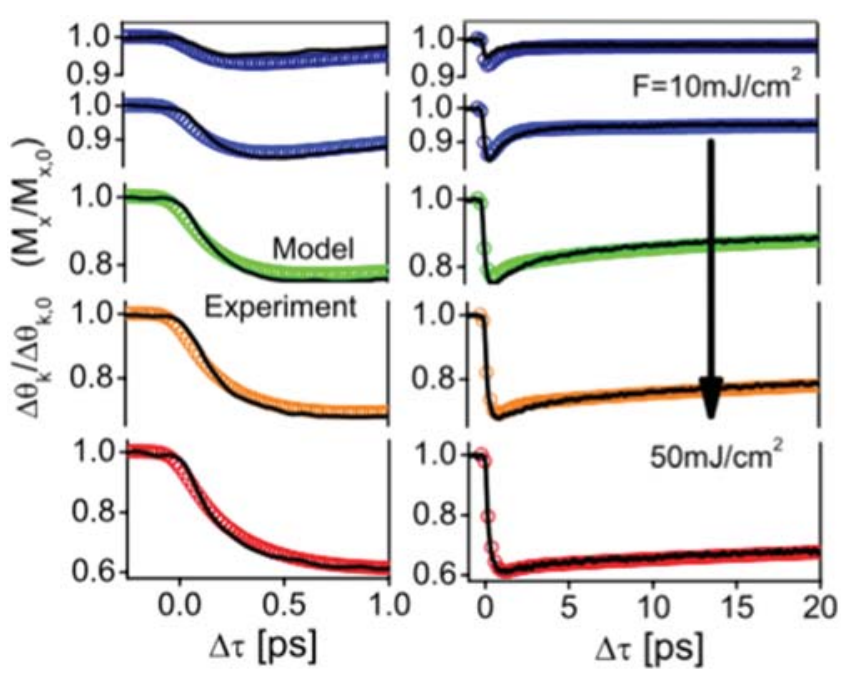

Figure 13. Sub-picosecond (left) and picosecond (right) magnetisation dynamics following the application of a femtosecond laser pulse for a $15 \mathrm{~nm} \mathrm{Ni}$ film. Comparison between the LLB model (symbols) and the experimental data (solid lines) for a range of laser pump fluence $F$. Reprinted figure with permission from [85], Copyright (2010) by the American Physical Society.

time scale of a few picoseconds. In order to describe the spin dynamics, the 2TM has been extended to a three-temperature model (3TM) [83]. Here, metallic ferromagnets are described in terms of three subsystems, electrons, phonons and spins, with individual heat capacities, temperatures and mutual interactions.

The description of the magnetisation dynamics in terms of a spin temperature has, however, to be questioned, since the spin subsystem might need much longer time scales to equilibrate [81]. For this reason, more sophisticated theories treat the spin dynamics microscopically as a spin model in a heat bath, where the heat-bath temperature is identified with the electron temperature, that can be calculated from a $2 \mathrm{TM}$ (equations (34) and (35)). While this approach was first realised with atomic spin models [81], later on the magnetisation dynamics was described by the LLB equation. In some of these works the effect of the laser was modelled as a simple square-like heat pulse rather than a temperature profile given by the 2TM [56]. Although these simulations give useful insights into the demagnetisation processes, for a direct and quantitative comparison to experiment one needs to resort to the 2TM.

Figure 13 shows the first direct comparison between the LLB equation - coupled to the $2 \mathrm{TM}$ - and experimental data on laser induced ultrafast magnetisation dynamics in $\mathrm{Ni}$ thin films [76]. Here, it was assumed that the electrons act as a heat bath for the spin system, providing a time dependent temperature, $T_{\mathrm{e}}(t)$, as provided by the integration of the 2TM. The 2TM parameters were extracted from the time dependence of the experimentally measured reflectivity. This combined experimental and theoretical work evidenced the importance of thermal effects in the laser induced demagnetisation dynamics in $\mathrm{Ni}$, in contrast to pure quantum-mechanically induced spin-flip mechanisms, as summarised recently by Illg et al [86], or the so-called superdiffusive spin currents [87]. The authors showed that the timescales of the demagnetisation 
and remagnetisation processes slowed down as the laser fluence increases (see figure 13). This behaviour revealed that the temperature dependence of the sub-picosecond demagnetisation time scale $\tau_{\text {demag }}$ is determined by the temperature dependence of the longitudinal susceptibility, $\widetilde{\chi}_{\|}$, which at zero field diverges at the critical temperature $T_{\mathrm{c}}$. Since $\tau_{\text {demag }} \sim \widetilde{\chi}_{\|}$ (see equation (13) in section 2.1), the demagnetisation processes shows critical slowing down.

The distinctive laser-induced sub-picosecond demagnetisation followed by picosecond remagnetisation dynamics has been classified as type I dynamics. Interestingly, Roth et al [88] reported experimental data for Ni showing that, by increasing the ambient temperature towards $T_{\mathrm{c}}$, type I dynamics transits to a two-step demagnetisation dynamics, a first sub-picosecond followed by a second picosecond demagnetisation process. This two-step demagnetisation dynamics has been termed type II dynamics. The demagnetisation in the rare earths $\mathrm{Gd}$ and $\mathrm{Tb}$ presents a type II dynamics [89]. Based on these observations, a classification of the dynamics of ferromagnets was introduced based on the ratio $T_{\mathrm{c}} / \mu_{0}$ : slow dynamics for low values and fast dynamics for high values [90]. Magnetic materials with low Curie temperature and high atomic magnetic moment are therefore expected to present slow dynamics, e.g. rare-earth metals.

In this context Sultan et al [91] investigated the ultrafast magnetisation dynamics of $\mathrm{Gd}(0001)$ as a function of the ambient temperature both by experimental means using the femtosecond time-resolved magneto-optical Kerr effect (MOKE), and theoretically by means of the quantum LLB equation in combination with the 2TM [92]. In that work, for the first time the quantum LLB equation with $S=7 / 2$ (spin of the seven unpaired f electrons) was coupled to two different heat baths, the conduction electrons $\left(T_{\mathrm{e}}\right)$ and the phonon system $\left(T_{\mathrm{ph}}\right)$. The longitudinal relaxation dynamics of such a model is given by

$\dot{m} / \gamma=\sum_{b} \alpha_{\|, \mathrm{b}}\left(T_{b}\right)\left\{\begin{array}{cc}\frac{1}{2 \widetilde{\chi}_{\|}}\left(1-\frac{m^{2}}{m_{\mathrm{e}}^{2}}\right) m, & T_{b} \lesssim T_{\mathrm{c}} \\ -\frac{1}{\widetilde{\chi}_{\|}}\left(1+\frac{3}{5} \frac{T_{\mathrm{c}}}{T-T_{\mathrm{c}}} m^{2}\right) m, & T_{b} \gtrsim T_{\mathrm{c}}\end{array}\right.$

where $T_{b} \equiv T_{\mathrm{e}}, T_{\mathrm{ph}}$. The damping parameters are given by equation (22), where the intrinsic damping parameters, $\lambda_{\mathrm{s}-\mathrm{e}}$ and $\lambda_{\mathrm{s}-\mathrm{ph}}$, were considered to depend on the different excitations of electrons and phonons.

Sultan et al found that at temperatures below the Debye temperature a hot-electron-mediated process can describe the experimentally found demagnetisation times. At higher temperatures, above $\theta_{\mathrm{D}}$, spin-phonon-mediated processes slow down the demagnetisation process, which can explain the observed longer demagnetisation times (see figure 14). Interestingly, it has been recently found that in Gd the orbital and spin angular momentum have rather disparate ultrafast dynamics and can be measured separately [93]. Atomistic spin model simulations reproduce experimental data nicely by assuming that the itinerant d-and localised f-electron spins are considered separately. This calls for the future use of the

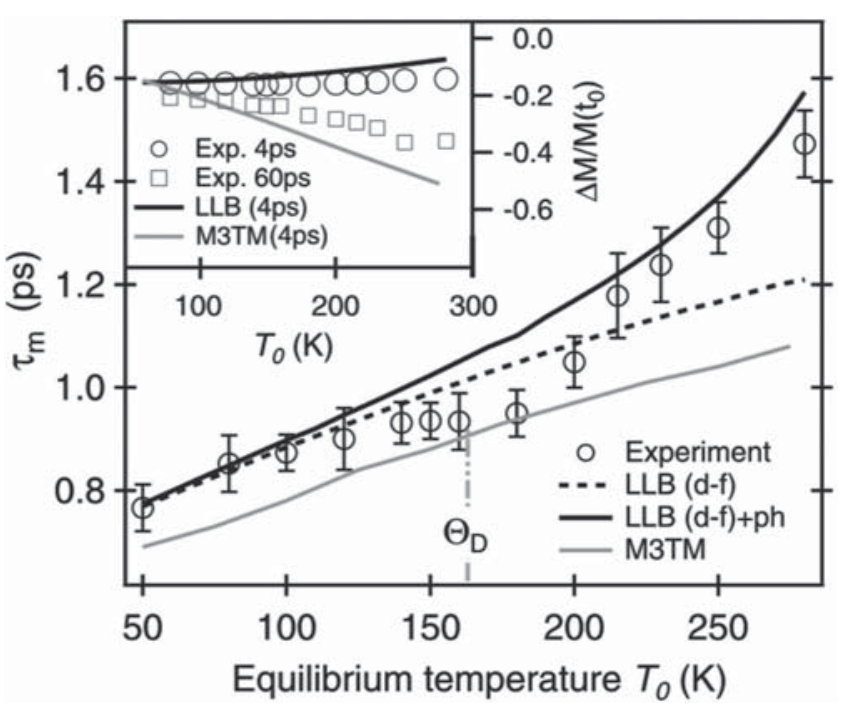

Figure 14. The demagnetisation time $\tau_{m}$ as a function of the ambient temperature $T_{0}$. Symbols represent the experimental data points, while lines represent the modelling results considering only electron-mediated spin flips (dashed) and combined electron- and phonon-mediated spin flips (solid line). The grey line represents the results obtained within the M3TM model assuming phononmediated spin-flip mechanisms. The inset shows $\Delta M / M_{0}$ at the indicated time delays. Reprinted figure with permission from [91], Copyright (2012) by the American Physical Society.

two-sublattice LLB equation (see section 2.2) to model the disparate dynamics of the localised and delocalised electron magnetisation dynamics in rare-earth metals.

More recently, the LLB equation has been used to resolve the role of the heated electrons in ultrafast spin dynamics of nanogranular $\mathrm{FePt} \mathrm{L1} 1_{0}$ thin films. Mendil et al [94] investigated the dynamics of FePt after application of laser pulses of a range of fluences. Notably, they found that the demagnetisation process transited from type I at low-to-intermediate fluence to type II in the high-fluence regime. Their simulations were based on the micromagnetic quantum LLB equation $(S=3 / 2)$ and the $2 \mathrm{TM}$. The parameters defining the $2 \mathrm{TM}$ parameters were inferred from the experimentally measured reflectivity, similar to the procedure followed in Ni thin films [85]. However, the set of parameters defining the $2 \mathrm{TM}$ was not uniquely determined, and two limiting possibilities were discussed, in terms of low and normal electron heat capacity. The authors found that the experimental data were theoretically reproducible when the specific heat of the electrons was taken with a rather low value for a transition metal like Fe. This reduction of the electron specific heat is attributed to the reduction of the density of states at the Fermi level owing to effects of alloying to Pt. The effect on the $2 \mathrm{TM}$ of a reduced $C_{\mathrm{e}}$ is that the electron temperatures last longer in the temperature region above $T_{\mathrm{c}}$, thereby promoting critical spin fluctuations that drive the transition from type I to type II. Figure 15 shows results of their simulations for a range of laser fluence. The transition from type I to type II at higher fluence could have strong implications for the use of lasers in $\mathrm{FePt} \mathrm{L}_{0}$ for so-called heat-assisted magnetic recording, and even for all-optical switching, as recently demonstrated experimentally by Lambert et al [5]. Recently, Klimling et al [95] 


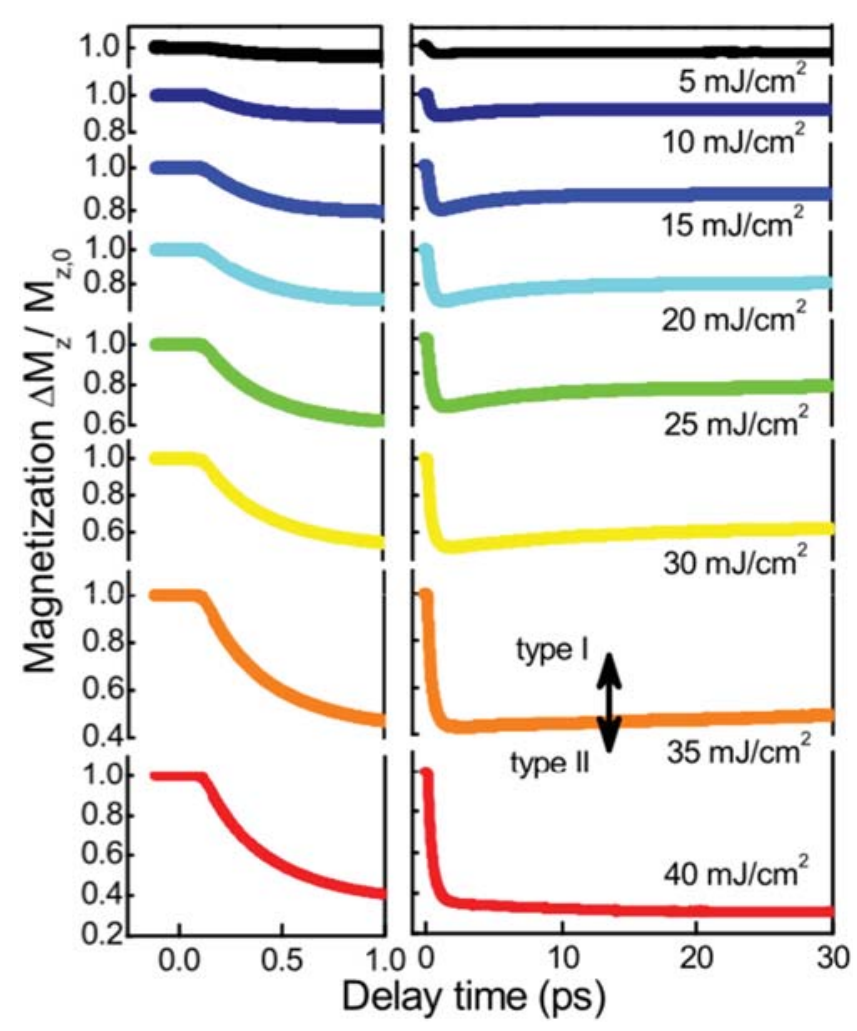

Figure 15. Demagnetisation dynamics of nanogranular FePt $\mathrm{L} 1_{0}$ thin films described by the LLB model. As the fluence of the laser pulse increases the dynamics transits from type I to type II. Reprinted with permission from [94], Copyright @ 2014, Rights Managed by Nature Publishing Group.

experimentally found a similar transition in $\mathrm{Cu}$-doped $\mathrm{FePt}$ thin films using picosecond laser pulses.

\subsection{Heat-assisted magnetic recording}

Further increase of magnetic storage density is limited by the so-called magnetic trilemma, where higher density requires smaller grain volumes with ensured thermal stability. Thermal stability is secured by using storage materials with high magnetic anisotropy. However, then their high coercive fields are a limiting factor for recording, as the maximum magnetic field produced by actual recording heads is limited by the magnetisation saturation of the pole. Heat-assisted magnetic recording (HAMR) has been proposed a possible solution to the magnetic trilemma [3]. HAMR utilises the temperature dependence of the anisotropy, which decreases with temperature. Therefore heating the material towards the Curie temperature will substantially decrease the energy barrier, and a fairly low magnetic field can reverse the magnetic state. Here, femtosecond laser pulses have been proposed as a fast way to heat magnetic materials to $T_{\mathrm{c}}$. However, close to $T_{\mathrm{c}}$ longitudinal magnetic fluctuations can have a significant impact on the expected energy barriers and therefore the relaxation time of the magnetisation, as we have discussed in the previous section for the case of FePt.

In this context the LLB equation has been an appropriate model to investigate the reversal modes of a ferromagnetic nanoparticles at temperatures close to $T_{\mathrm{c}}$. Kazantseva et al
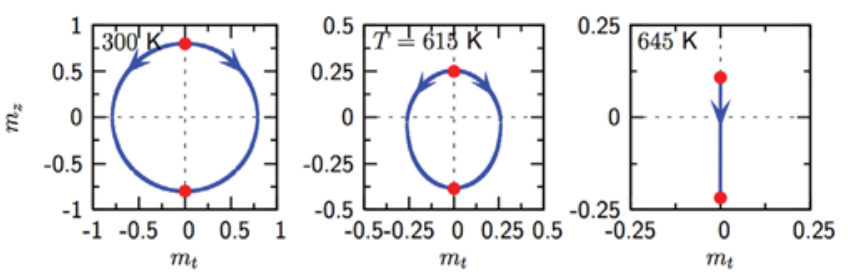

Figure 16. Reversal modes of an FePt nanoparticle: At low temperature, $T=300 \mathrm{~K}$, the reversal is nearly circular (Stoner-Wohlfarth type). At higher temperatures, $T=615 \mathrm{~K}$, the reversal mode is elliptical. Close enough to $T_{\mathrm{c}}=650 \mathrm{~K}$, at $T=645 \mathrm{~K}$, the reversal is linear. The red points represent the stationary points, which are the initial condition as well as the final state. Reproduced from [57]. Copyright (c) EPLA, 2009.

[57] investigated thermally assisted switching based on the LLB equation. Analytical expressions for the reversal times as a function of both the temperature and external magnetic field were calculated. Three reversal paths were found depending on the temperature regime (see figure 16). Circular reversal, where the magnetisation length is conserved during the reversal process, is strictly only possible at zero temperature. At any finite temperature during the reversal process the effective field acting on the magnetisation $\mathbf{m}$ is not constant, and hence the magnetisation is not conserved. This makes the reversal path elliptical rather than circular. At some critical temperature below $T_{\mathrm{c}}$, the reversal becomes linear. The linear reversal mode is a fundamentally different process, where the magnetic order is destroyed before it starts to build up in the opposite direction without any transverse component. Since this reversal is associated with the exchange interaction it is much faster than circular and elliptical reversal paths.

Later on, Barker et al [96] compared the analytical expressions derived by Kazantseva [57] to atomistic spin model simulations (see figure 17). Similarly, for nanograins Ellis and Chantrell investigated the role of nanoscale effects in the switching behaviours by varying the nanograin size down to $2 \mathrm{~nm}$, using both the LLB and atomistic spin simulations [97]. The agreement between the LLB and atomistic simulations in both works was excellent, which serves as another validation of the LLB equation. Also, Greaves et al [98] have presented an alternative version to the quantum LLB by directly modifying the classical LLB equation. They investigated the reversal times for HAMR processes in $8 \mathrm{~nm}$ nanoparticles. Overall, one can conclude that HAMR could become a reliable recording scheme for highly anisotropic and thermally stable media with reduced nanograin size. Therefore, it is expected that computer simulations using the LLB equation will become an important part of the design process [99-101] of the next generation storage media.

Another alternative class of material to be used in HAMR technology is composed of nanograins with graded anisotropy and Curie temperature, the simplest example being a bilayer composed of hard (high $T_{\mathrm{c}}$ ) and soft (low $T_{\mathrm{c}}$ ) magnetic material. This grading of the magnetic properties helps to reduce the field needed to reverse the magnetisation. Vogler et al [102] utilised the stochastic LLB equation to calculate the thermal stability of this kind of bilayer. Within a multi-scale approach, 


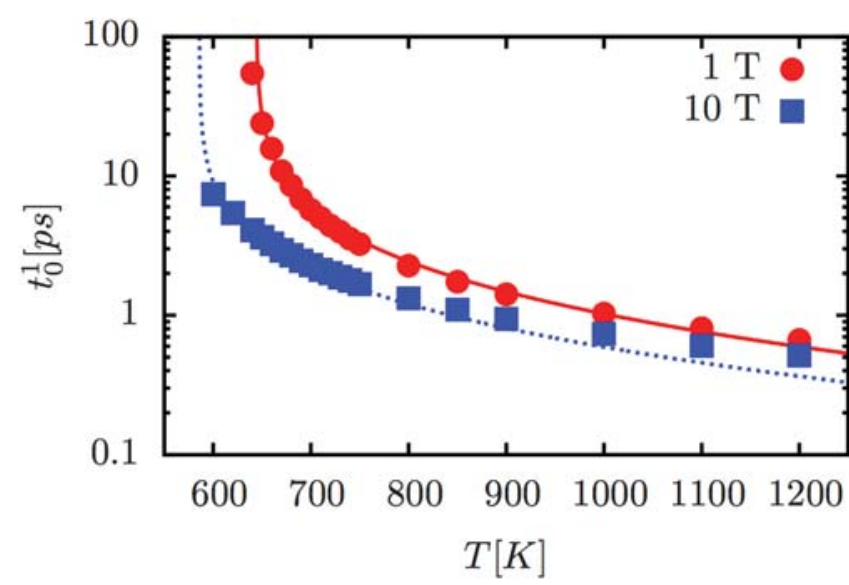

Figure 17. A comparison of the characteristic reversal time $t_{0}^{1}$ as a function of temperature, through $T_{\mathrm{c}}$, in a $6 \mathrm{~nm}$ cube of FePt, $T_{\mathrm{c}}=660 \mathrm{~K}$. Two magnetic fields along the $z$ axis—opposing the magnetisation - of 1 and $10 \mathrm{~T}$ are compared. Atomistic spin model simulations (symbols) and the analytic solution of the LLB equation (solid lines) are from [57]. Reprinted with permission from [96]. Copyright (2010), AIP Publishing LLC.

similar to that presented in section 3.1, the input parameters of the hard and soft materials were calculated using atomistic spin dynamics simulations. Here, a new susceptibility was introduced, the susceptibility of the magnetisation modulus, which was used instead of the longitudinal susceptibility. Furthermore, an expression for the micromagnetic exchange parameter coupling the two distinct magnets was suggested,

$$
A(T)=A_{\mathrm{i}, \mathrm{ex}}(0) \sqrt{m^{\alpha}(T) m^{\beta}(T)} .
$$

Here $A_{\mathrm{i}, \mathrm{ex}}(0)$ is an interlayer micromagnetic exchange stiffness and $\alpha$ and $\beta$ the scaling exponents of the micromagnetic exchange, as already discussed in section 3.1. The value of $A_{\mathrm{i}, \mathrm{ex}}(0)$ depends strongly on the characteristic length scales of the layers. Therefore, it was also estimated within a multiscale framework. The authors calculated switching probabilities under the influence of a Gaussian heat pulse and an external homogeneous magnetic field (see figure 18). The excellent agreement between the proposed LLB model for graded media and atomistic spin model simulations validated the author's approach.

\subsection{All-optical magnetisation switching}

Switching the magnetisation with ultrashort laser pulses is attractive for potential information storage device applications. The term all-optical switching (AOS) refers to the fact that some magnetic materials can be switched solely by the effect of a femtosecond laser pulse, without any applied magnetic field involved. For applications, the so-called helicity-dependent switching, where the orientation of the written magnetisation spot is set by the helicity of the incoming circularly polarised laser beam, is most promising. This effect was demonstrated for ferrimagnets [103, 104] but later also for layered, synthetic ferrimagnets [4] and recently even for ferromagnets [5]. A full understanding of the variety of effects which were found experimentally is still lacking. One possible

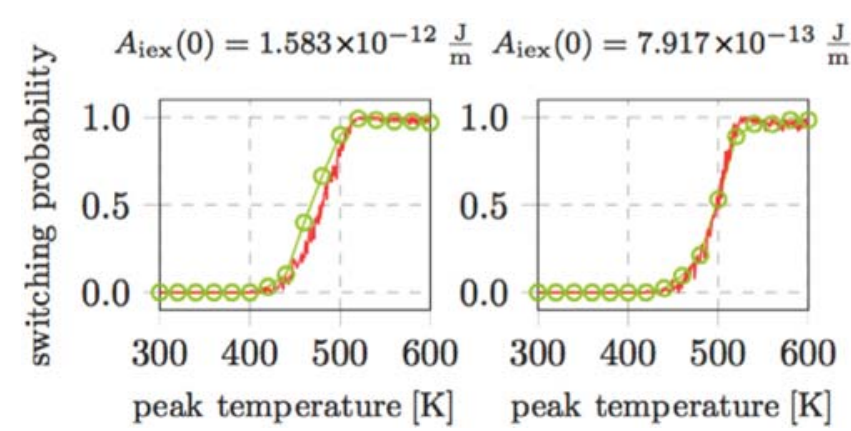

Figure 18. Comparison of atomistic switching probability curves (green lines with circles) with the results of the coarse-grained LLB model (red solid lines) for different inter-grain exchange constants. The investigated high/low $T_{\mathrm{c}}$ grain is subject to a Gaussian heat pulse with $t_{\text {pulse }}=100 \mathrm{ps}$ and an external field with $0.5 \mathrm{~T}$ strength. Reprinted figure with permission from [102], Copyright (2014) by the American Physical Society.

explanation for helicity-dependent AOS is, however, that the laser pulse induces some magnetisation along the direction of light caused by the so-called inverse Faraday effect.

To support this, single macro-spin simulations within the framework of the LLB equation were performed [104, 105]. The strong laser pulse heats the material, which was taken into account via the two-temperature model as described in the previous sections. The inverse Faraday effect was modelled as an effective field pulse. Though the material under investigation was the ferrimagnet $\mathrm{GdFeCo}$, the authors used the ferromagnetic LLB equation as an approximation. It was assumed that the inverse Faraday effect produces magnetic fields as strong as $20 \mathrm{~T}$ and that these fields last longer than the laser pulse itself. Under these assumptions it was shown that field pulse durations as short as 250 fs can be sufficient to reverse the magnetisation. Furthermore, it was found that the magnetisation switching occurs via a linear pathway [57] without any precession, as discussed above. Figure 19 shows a central result, comparing theory with experiment: only for a narrow range of laser powers is deterministic switching achievable.

The experimental observation of element-specific magnetisation dynamics in ferrimagnetic alloys started by employing ultrafast excitation in combination with the femtosecondresolved X-ray magnetic circular dichroism (XMCD) technique [106]. An astonishing example of such element-specific ultrafast magnetisation dynamics was first measured on ferrimagnetic GdFeCo alloys by Radu et al [9]. There, it was observed that the Gd demagnetises in around $1.5 \mathrm{ps}$, whereas the transition-metal FeCo sublattice has a much shorter demagnetisation time of 300 fs. Importantly, the switching was preceded by a novel non-equilibrium state where the magnetisations of both sublattices were pointing in the same direction for some picoseconds before complete reversal, the so-called transient ferromagnetic-like state. Ostler et al [8] showed both numerically_using atomistic spin models-and experimentally that ultrafast heating alone is a sufficient stimulus for the magnetisation reversal in GdFeCo alloys.

Using insights from LLB-based simulations for ferrimagnets as presented in section 2.2, and in combination with atomistic spin model simulations, Atxitia et al shed some 


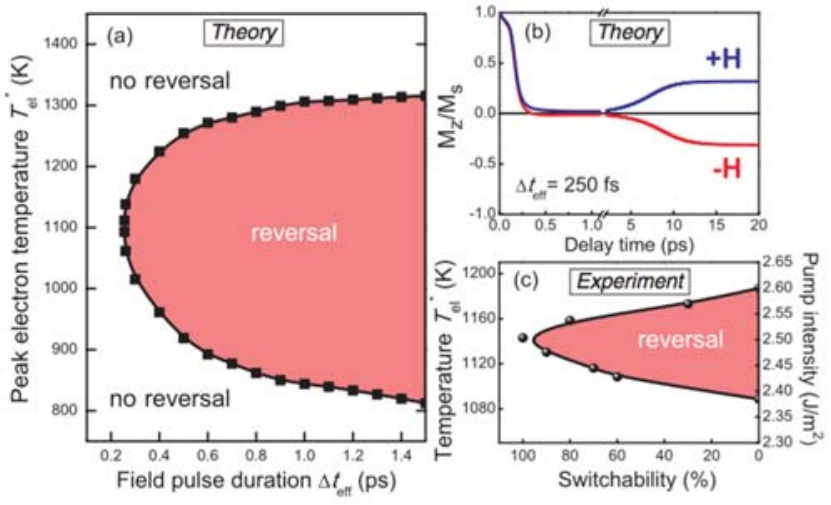

Figure 19. Phase diagram showing the magnetic state achieved within $10 \mathrm{ps}$ after the action of the optomagnetic pulse with IFE field strength $H_{\text {eff }}=20 \mathrm{~T}$ for different durations of the IFE pulse $t_{\mathrm{eff}}$ and peak electron temperatures $T_{\mathrm{el}}$. (b) The averaged $z$ component of the magnetisation versus delay time as calculated for $250 \mathrm{fs}$ magnetic field pulses for $H_{\mathrm{eff}}=20 \mathrm{~T}$ and $T_{\mathrm{el}}=1130 \mathrm{~K}$. (c) Measured switchability versus pump intensity for $\mathrm{Gd}_{22} \mathrm{Fe}_{68} \mathrm{Co}_{9}$ at room temperature. Reprinted figure with permission from [104], Copyright (2009) by the American Physical Society.

light on the understanding of this so-called thermally induced magnetisation switching in $\mathrm{GdFeCo}$, the temperature dependence of the transient ferromagnetic-like state [64], the reversal paths [107], and the role of the phonons [108]. Along the same line, a recent work by Suarez et al [109] investigated the demagnetisation times of $\mathrm{GdFeCo}$ alloys for a range of $\mathrm{Gd}$ concentration. The magnetic exchange parameters were varied for a better understanding of the effect of the demagnetisation times on the ability to switch. Further investigations using the LLB equation for ferrimagnets were made by Oniciuc et al [110]. The authors dealt with the dependence of the switching behaviour on the damping parameter and the $\mathrm{Gd}$ concentration. Extensive computer simulations allowed them to calculate a phase diagram of the reversal probabilities as a function of damping and Gd concentration. Nieves et al [111] have utilised the stochastic LLB equation to investigate the switching conditions under which the ferromagnet FePt shows AOS under the action of a heat pulse assisted by a constant or opto-magnetic field (coming from the IEF). They concluded that the magnitudes of the opto-magnetic field might be too large for real situations, calling for further experiments and theoretical investigations of the origin of the inverse Faraday effect in FePt.

The field of AOS is a rapidly growing research area, promising new and faster ways to control magnetically stored information. AOS has even been demonstrated in nanogranular FePt $\mathrm{L}_{0}$ thin films[5]. Still, many open questions exist calling for further experimental and theoretical work wherebecause of the heating effects-the LLB equation will be of utmost importance.

\subsection{Domain walls: finite temperature, spin torques and temperature gradients}

Well controlled domain walls could become important constituents of future magneto-electronic devices [112]. Soon

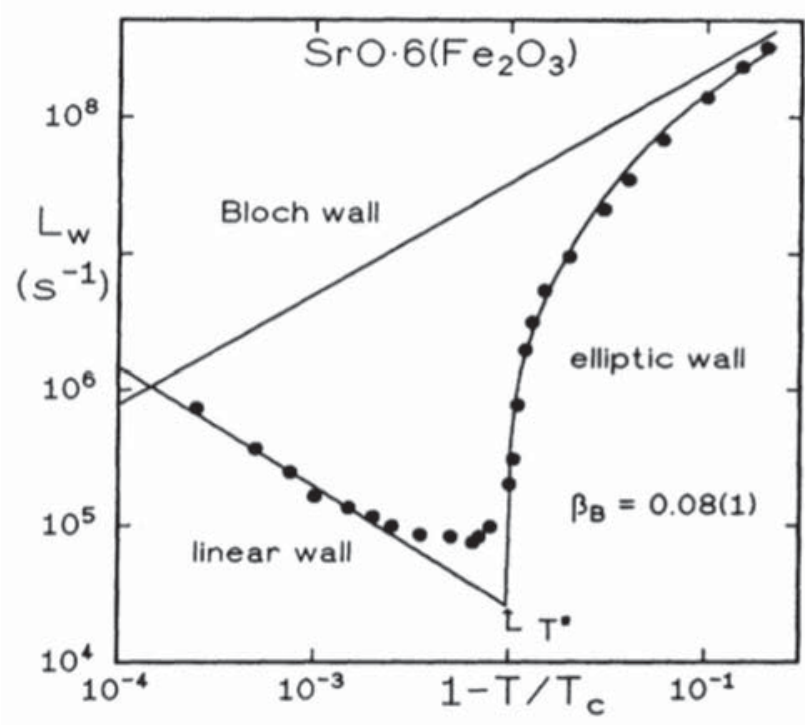

Figure 20. Temperature variation of the kinetic coefficient of the wall relaxation $L_{\omega}$. Solid lines correspond to fits of the linear wall $\left(T>T^{\star}\right)$ and elliptical wall dynamics $\left(T<T^{\star}\right)$. Reprinted figure with permission from [114], Copyright (1993) by the American Physical Society.

after the derivation of the LLB equation it was realised that elevated temperatures will affect domain walls (DWs), regarding their structure and their dynamics.

First, within the framework of Ginzburg-Landau theory it was shown that for a one-dimensional domain wall profile (e.g. a Bloch wall) the easy-axis and hard-axis components of the magnetisation, respectively, are two separate order parameters with different critical temperatures [113]. The perpendicular magnetisation component which arises necessarily in a domain wall has at finite temperatures values lower than the easy-axis equilibrium magnetisation, leading to so-called 'elliptical domain walls'. For a temperature $T_{\mathrm{h}}$ which is lower than the Curie temperature $T_{\mathrm{c}}$ of the bulk material, the perpendicular component even vanishes completely, leading to the so-called 'linear domain walls' for temperatures $T_{\mathrm{h}}<T<T_{\mathrm{c}}$. Garanin used the LLB equation to investigate the dynamics of elliptical and linear domain walls further [42]. New effects for the dynamics of the DWs were found, which could be compared to experiments. This was the first experimental verification of the validity of the LLB approach [114, 115]. Figure 20 shows a comparison between experimental measurement of the DW relaxation coefficient $L_{\omega}$ and the LLB model. The transition from elliptical to linear walls occurs at $T^{\star}=0.99 T_{\text {c }}$.

Current-induced domain wall motion has been suggested as an alternative route to induce switching avoiding external magnetic fields. While current-induced domain wall motion is experimentally well established $[116,117]$, the underlying physical mechanisms are not completely understood, especially the importance of the adiabatic and the non-adiabatic spin torque terms $[118,119]$ and the influence of temperature on the wall dynamics.

To theoretically predict the behaviour of a spin texture under current, one can numerically solve the LL equation. Spin torque effects are then taken into account by including 


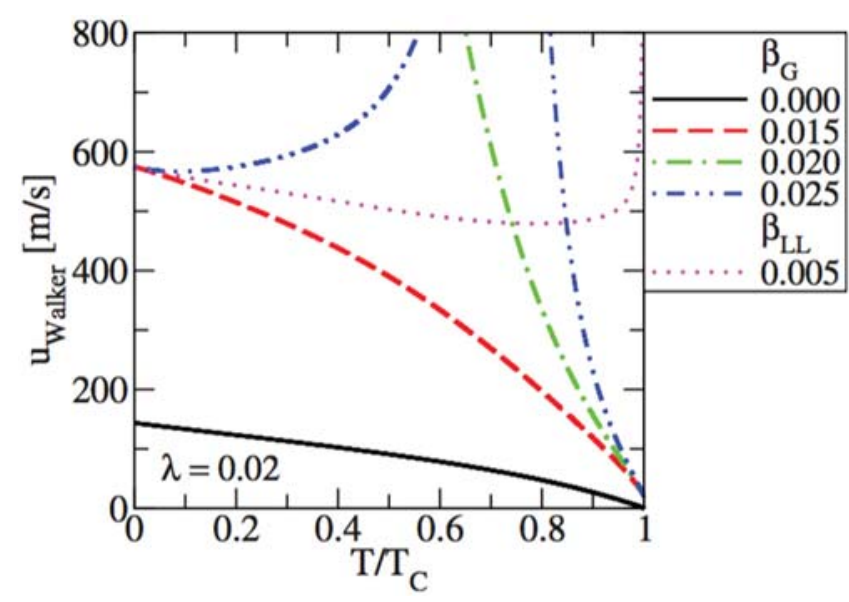

Figure 21. Walker threshold $u_{\text {Walker }}$ versus the reduced temperature $T / T_{\mathrm{c}}$ for different values of the non-adiabatic pre-factor $\beta_{\mathrm{G}}$ as well as $\beta_{\mathrm{LL}}$. Reprinted figure with permission from [122], Copyright (2009) by the American Physical Society.

the adiabatic and the non-adiabatic torque terms [118-121]. However, conventional micromagnetic calculations for larger system sizes lack the correct description of temperature effects because of the assumption of a constant magnetisation length. An alternative approach here is again the LLB equation including the above mentioned spin torque terms.

The first paper using this approach was by Schieback et al [122]. In this paper, DW motion was studied where the LLB equation of motion was extended by adding the spin torque terms

$$
\mathbf{T}=-u_{x} \frac{\partial \mathbf{m}}{\partial x}+\frac{\beta}{m} \mathbf{m} \times u_{x} \frac{\partial \mathbf{m}}{\partial x} .
$$

Here $u_{x}=P^{0} j_{e} \mu_{\mathrm{B}} / M_{\mathrm{s}}^{0} e$ parametrises the spin current with $P^{0}$ the polarisation of the current, $j_{e}$ the electric current density, $e$ the charge of the electron, $M_{\mathrm{s}}^{0}$ the saturation magnetisation at $T=0 \mathrm{~K}$, and $\mu_{\mathrm{B}}$ Bohr's magneton. $\beta$ is the so-called nonadiabaticity factor, a parameter the details of which are still under debate.

One of the advantages of the LLB formulation is that it allows for analytical calculations. Schieback et al [122] were able to calculate the velocity of a DW wall as a function of the spin-polarised current $u_{x}$. Below the Walker threshold the DW velocity is

$$
v_{\mathrm{DW}}=\frac{\beta m_{\mathrm{e}} u_{x}}{\alpha_{\perp}}
$$

where the Walker threshold is given by

$$
u_{\text {Walker }}=\frac{\gamma}{4 \widetilde{\chi}_{\perp}} \Delta_{\text {Walker }} \frac{\alpha_{\perp} m_{\mathrm{e}}}{\left|\alpha_{\perp}-\beta m_{\mathrm{e}}\right|} .
$$

Above the Walker threshold analytical expressions for the DW velocity were also calculated [122]. Because of the temperature dependence of the material parameters in the LLB approach and the interplay between the adiabatic and the nonadiabatic spin torque, the resulting onset of the Walker breakdown was found to be very sensitive to the temperature (see figure 21).

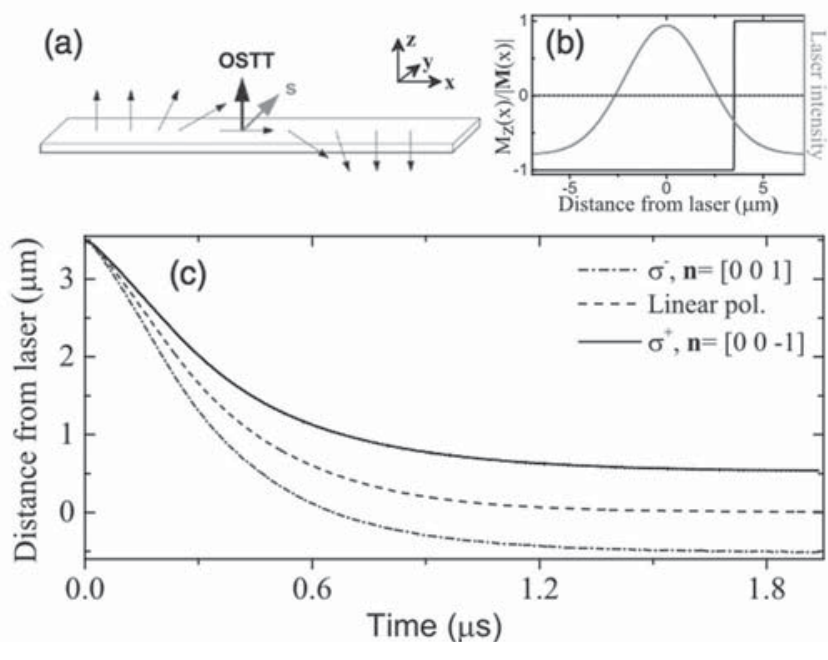

Figure 22. (a) Schematic diagram of the direction of the optical spin-transfer torque (OSTT) acting on the magnetisation at a Néel domain wall (DW). (b) Initial position of the DW and the laser spot intensity profile. (c) DW motion following the application of an 80 $\mathrm{MHz}$ train of laser pulses. For linear polarisation, the DW moves to the centre of the hot spot created by the laser heating. For circular polarisation, $\sigma^{ \pm}$, the additional spin-transfer torque slows down (speeds up) the DW motion. Reprinted figure with permission from [125], Copyright (2015) by the American Physical Society.

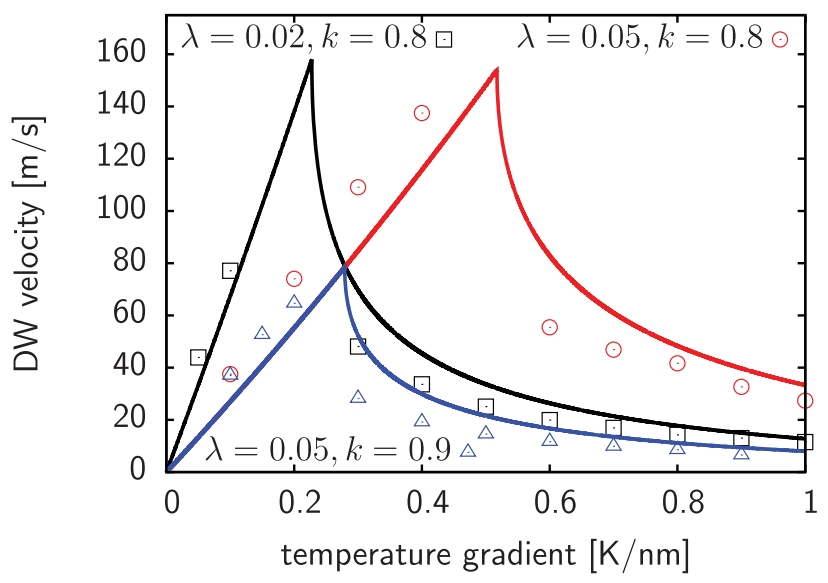

Figure 23. DW velocity versus temperature gradient for two different damping constants $\lambda$. Numerical data are compared with an analytical expression. (Taken from [128].)

Haney and Stiles [123] proposed a similar LLB equation with an additional Slonczewski term. The authors compared the resulting LLB equation to atomistic spin model simulations, and a good agreement was found. Oniciuc et al [124] extended the approach of Schieback et al to include the angular dependence of the spin-transfer parameters, as originally proposed by Slonczewski.

Recently, Ramsay et al [125] studied optical manipulation of DWs in the prototypical dilute magnetic semiconductor GaMnAs. They experimentally investigated the DW motion after the application of laser pulses with two circular helicity $\sigma^{ \pm}$as well as linear $\sigma^{0}$. The DW was created far from the laser spot. The experimental observations were well described by the LLB equation (see figure 22). For linear polarised light, $\sigma^{0}$, the effect of the laser is just to create a temperature 


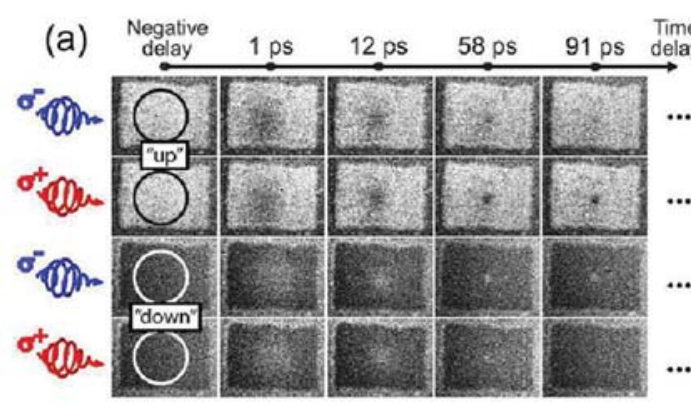

(c)

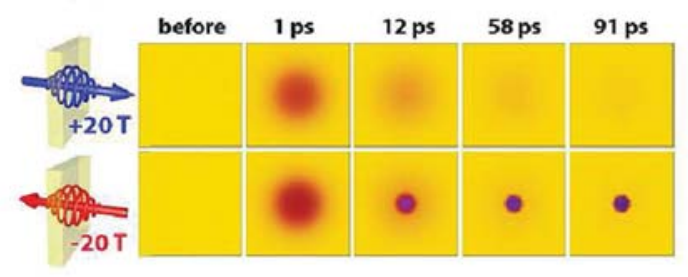

(b)

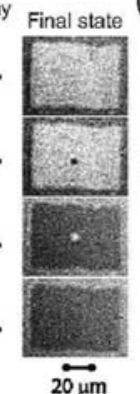

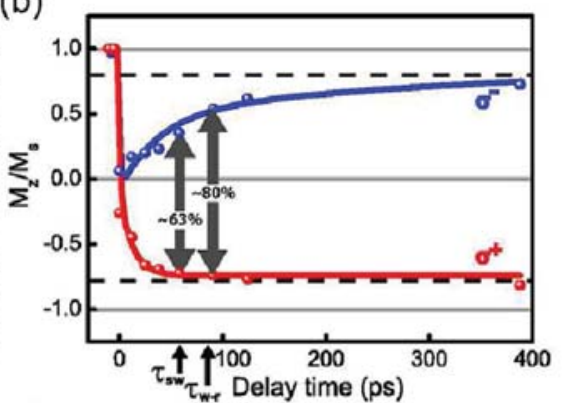

(d) 1

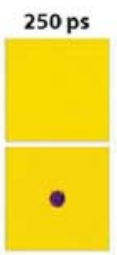

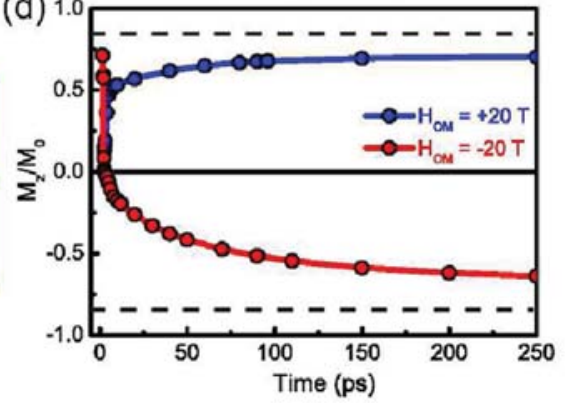

Figure 24. (a) The magnetisation evolution in $\mathrm{Gd}_{24} \mathrm{Fe}_{66.5} \mathrm{Co}_{9.5}$ after excitation with $\sigma^{+}$and $\sigma^{-}$circularly polarised pulses at room temperature. The film is initially magnetised up (white domain) or down (black domain). The last column shows the final magnetic state of the film after a few seconds. The circles show areas where the effect of the laser pulse on the magnetic state is detected within the sensitivity of the setup. Note that the pump spot size is 50-70 $\mu \mathrm{m}$ and larger than the images. (b) The averaged magnetisation in the switched areas $(\sim 5 \mu \mathrm{m})$ after $\sigma^{+}$and $\sigma^{-}$laser pulses (c) Distributions of the $z$ component of the magnetisation across the $10 \mu \mathrm{m} \times 10 \mu \mathrm{m} \times 5 \mathrm{~nm}$ ferromagnetic film at different time delays after the combined action of a $100 \mathrm{fs}$ long laser pulse and a $250 \mathrm{fs}$ long opto-magnetic field. Reprinted figure with permission from [105], Copyright (2012) by the American Physical Society.

profile. The DW wall moves to the hotter region created by the laser spot. The circularly polarised light however excites a net density of photo-induced spin carriers $\mathbf{s}$. This $\mathbf{s}$ exerts a spin-transfer torque on the magnetisation via the exchange interaction that moves the DW. To describe this effect, the authors extended the LLB equation by adding an extra field representing the optical spin transfer torque (OSST), $\mathbf{H}_{\mathrm{eff}}^{\mathrm{OSTT}}=J_{\mathrm{eff}} / \mu_{0} M_{\mathrm{e}}(T) \mathbf{s}$, where $J_{\text {eff }}$ is the exchange interaction between carriers and localised magnetic moments, and $M_{\mathrm{e}}(T)$ is the equilibrium saturation magnetisation. At the same time the evolution of $\mathbf{s}$ was calculated via an auxiliary equation of motion: $\dot{\mathbf{s}}=A \mathbf{m} \times \mathbf{s}+R(t) \hat{n}-\mathbf{s} / \tau_{c}$, where $A=J_{\text {eff }} / m_{\mathrm{eq}} \hbar$, and $R(t)$ is the rate of laser spin pumping and $\tau_{c}$ the spin relaxation time.

In [126] Hinzke and Nowak demonstrated the existence of thermally driven DW motion in a temperature gradient by computer simulations based on an atomistic spin model as well as on the LLB equation of motion. A thermodynamic explanation for this kind of DW motion rests on the minimisation of the free energy of the DW. For a DW at finite temperature, the free energy is $\Delta F(T)=\Delta U-T \Delta S$, where $\Delta U$ is the internal energy and $\Delta S$ the entropy of the DW. The free energy of the DW can be calculated from the difference of the internal energy between systems with and without the DW [75]. For a ferromagnetic system, it is a monotonically decreasing function of temperature, which goes along with the fact that the entropy is a monotonically increasing function of temperature. This rather general argument explains a DW motion towards the hotter parts of the sample where the free energy is lowered and the entropy increases [127-129]. Furthermore, it has been shown by Schlickeiser et al that the DW motion is caused by a so-called entropic torque, similar to the spin transfer torque that acts due to a spin polarised current. The exchange stiffness is weaker for higher temperature and therefore an effective torque on the DW is created, driving it towards the hotter region [128]. In the one-dimensional limit an analytical formula was derived for the speed of the DW. Below the Walker threshold Schlickeiser et al proposed

$$
v_{\mathrm{D}}=-\frac{2 \gamma}{M_{\mathrm{s}}} \frac{1}{\alpha_{\perp}} \frac{\partial A}{\partial T} \frac{\partial T}{\partial z}
$$

where the driving force is the temperature gradient in connection with the temperature dependence of the exchange stiffness $A(T)$. Figure 23 shows the DW velocity as calculated from the LLB approach and compares an analytical formula with numerical data.

\subsection{Thermal influence on domain structures: large scale simulations}

The strength of the LLB equation lies in the fact that-in contrast to atomistic spin model simulations-large scale samples can be treated numerically, so the influence of thermal excitations on domain structures can be investigated.

In [105] a sample size of $10 \mu \mathrm{m} \times 10 \mu \mathrm{m}$ with $5 \mathrm{~nm}$ resolution was used to investigate how the laser spot writes a domain all-optically in FeGd (see section 4.3). The dipole-dipole interaction was taken into account rigorously using FFT 


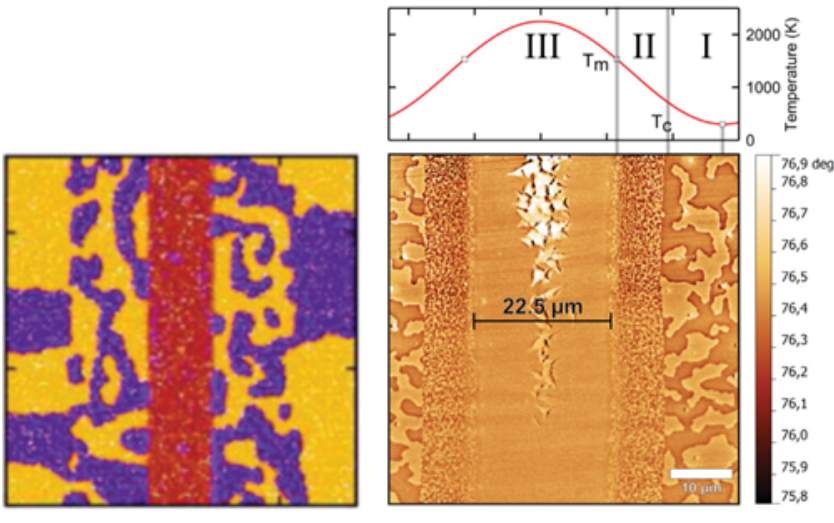

Figure 25. Right: different regions for the magnetic structure using an interference period of $55 \mu \mathrm{m}$ as well as the calculated temperature distribution. Left: simulated results for the magnetic configuration assuming a reduced anisotropy $(65 \%)$ and $20 \%$ defects. Reproduced from [130]. (C) IOP Publishing Ltd.

methods. The film was subject to a heat pulse, calculated with a two-temperature model, and an inverse Faraday field pulse with Gaussian spatial profile with radius $r_{0}=2.1 \mu \mathrm{m}$. The calculated and measured distributions of the magnetisation at various time delays are shown in figure 24. A comparison of the calculated and experimental results shows that the spatial profile of the process of the relaxing magnetic state is determined by the spatial distribution of the laser pulse intensity, which defines the distribution of both the electronic temperature and the opto-magnetic field.

A further direct comparison of simulations of the LLB equation with experiments was based on nanosecond pulsed two-beam laser interference, which was used to generate two-dimensional temperature patterns on a magnetic thin film sample [130]. Experimentally, Stärk et al demonstrated that the original domain structure of a $\mathrm{Co} / \mathrm{Pd}$ multilayer thin film changes drastically upon exceeding the Curie temperature by thermal demagnetisation (region II in figure 25). At even higher temperatures the multilayer system is irreversibly changed (region III). In this area no out-of-plane magnetisation can be found after a subsequent $\mathrm{AC}$ demagnetisation. These findings were supported by numerical simulations of the LLB equation, which showed the importance of defect sites and anisotropy changes to model the experiments. Thus, a one-dimensional temperature pattern could be transformed into a magnetic stripe pattern. In this way one can produce magnetic nanowire arrays with lateral dimensions of the order of $100 \mathrm{~nm}$.

\subsection{Other applications}

This review cannot account for all applications of the LLB equation that can be found in the literature, but in this section will at least briefly mention further work where the LLB equation was exploited. With its temperature dependent parameters the LLB equation allows for the calculation of temperature dependent phenomena which so far had only been investigated at low or constant temperature.

One example is ferromagnetic resonance, where the LLB equation was used by Ostler et al [131] to derive analytic expressions for temperature-dependent absorption spectra as probed by ferromagnetic resonance. By constructing a multimacrospin model the study was extended to investigate the effects on the damping and resonance frequency in micrometre-sized structures. Similar calculations were performed by Lebecki [132].

Another advantage of the LLB equation is that-since the magnitude of the magnetisation is not conserved-it avoids singularities, e.g. Bloch points. In this context Lebecki et al investigated vortex core dynamics and Bloch points at elevated temperatures using the LLB equation in connection with a micromagnetic framework for permalloy [133-135]. This framework enables micromagnetic modelling of a Bloch point avoiding the problem of singularities, which have been reported in the literature so far. Relevant properties of the vortex core, such as its radius, the magnetisation drop in its centre, and the radius of this magnetisation drop were extracted. The dependence of the vortex core radius on temperature agrees well with the theoretical predictions, if only temperature dependent parameters are taken into account. Switching in thin circular permalloy discs caused by the application of a slowly increasing magnetic field oriented orthogonally to the disc was also considered. In particular, the switching field went to zero at a significantly smaller temperature than the Curie temperature, and the deduced nucleation volume was smaller than the typical grain size in permalloy.

\section{Summary and outlook}

The theoretical description of magnetisation dynamics and magnetic textures under conditions where thermal excitations dominate is of utmost importance in modern magnetism. The LLB equation offers a framework for both analytical and numerical treatment of a broad variety of problems. Originally intended for the modelling of ferromagnetic materials, it has been extended to magnetic materials with two sublattices, e.g. antiferromagnets and ferrimagnets. Classical as well as quantum versions of the LLB equation exist, and a stochastic version has been derived as well to include explicit thermal fluctuations where necessary.

The numerical effort when solving the LLB equation is comparable to that for more conventional methods in micromagnetism which are based on the LL equation. The main problem currently is that the LLB equation is based not on a set of material parameters, but rather on a set of thermodynamic functions. These functions, mostly the temperature dependence of exchange stiffness, susceptibilities, and spontaneous magnetisation, have to be known in advance. In the easiest case they follow from a mean-field calculation for a more or less realistic spin model describing the material that has to be modelled. So far more detailed calculations have followed from either Monte Carlo simulations or microscopic simulations of the stochastic LL equation. However, other methods which yield thermal equilibrium properties can also be taken into account, including random phase approximation and quantum Monte Carlo, but also fitting to experimental data.

The LLB equation is hence also an important part of multiscale modelling efforts which link $a b$ initio methods with 
spin models and-on larger length scales-with mesoscopic theories. Consequently, a broad range of applications exists, ranging from laser-induced spin dynamics to spintronics and spin caloritronics. In all these research areas thermal excitations are relevant, originating either from the heating effect of the laser, from Joule heating via the applied currents, or from thermal gradients that are applied to exploit magnonic spin currents. Here, exploiting the LLB equation has already considerably contributed to the understanding of new phenomena, and more research efforts along these lines can be expected for the future.

\section{Acknowledgment}

We thank the DFG for financial support through the SFB 767 and SPP 1538 as well as the Center of Applied Photonics at the University of Konstanz. UA gratefully acknowledges support from EU FP7 Marie Curie Zukunftskolleg Incoming Fellowship Programme, University of Konstanz (Grant No. 291784).

\section{References}

[1] Boulle O, Malinowski G and Kläui M 2011 Mater. Sci. Eng. R 72187

[2] Ralph D C and Stiles M D 2007 J. Magn. Magn. Mater. 3201190

[3] Kryder M, Gage E, McDaniel T, Challener W, Rottmayer R, Ju G, Hsia Y and Erden M 2008 IEEE Proc. 961810

[4] Mangin S et al 2014 Nat. Mater. 13286

[5] Lambert C H et al 2014 Science 3451337

[6] Kirilyuk A, Kimel A V and Rasing T 2013 Rep. Prog. Phys. 76026501

[7] Kirilyuk A, Kimel A V and Rasing T 2010 Rev. Mod. Phys. 822731

[8] Ostler T A et al 2012 Nat. Commun. 3666

[9] Radu I et al 2011 Nature 472205

[10] Bauer G E W, Saitoh E and van Wees B J 2012 Nat. Mater. 11391

[11] Landau D L and Lifshitz E 1935 Phys. Z. Sowjetunion 8153

[12] Gilbert T L 1955 Phys. Rev. 1001243

[13] Brown W F 1963 Micromagnetics (New York: Wiley)

[14] Heisenberg W 1928 Z. Phys. 49619

[15] Lakshmanan M 2011 Phil. Trans. R. Soc. A 3691280

[16] Wieser R 2011 Phys. Rev. B 84054411

[17] Qu T and Viictora R H 2014 J. Appl. Phys. 11517

[18] Mankovsky S, Ködderitzsch D, Woltersdorf G and Ebert H 2013 Phys. Rev. B 87014430

[19] Liu Y, Yuan Z, Wesselink R, Starikov A and Kelly P 2014 Phys. Rev. Lett. 113207202

[20] Udvardi L, Szunyogh L, Palotás K and Weinberger P 2003 Phys. Rev. B 68104436

[21] Ebert H and Mankovsky S 2009 Phys. Rev. B 79045209

[22] Kronmüller H and Fähnle M 2003 Micromagnetism and the Microstructure of Ferromagnetic Solids (Cambridge: Cambridge University Press)

[23] Donahue M J and Porter D G 1999 OOMMF User's Guide Technical Report 6376 (http://math.nist.gov/oommf)

[24] Lyberatos A, Berkov D V and Chantrell R W 1993 J. Phys.: Condens. Matter 58911

[25] García-Palacios J L and Lázaro F J 1998 Phys. Rev. B 5814937

[26] Coffey W T, Kalmykov Y P and Waldron J T 2004 The Langevin Equation: with Applications to Stochastic
Problems in Physics, Chemistry and Electrical Engineering (World Scientific Series in Contemporary Chemical Physics vol 14) 2nd edn (Singapore: World Scientific)

[27] Antropov V P, Tretyakov S V and Harmon B N 1997 J. Appl. Phys. 813961

[28] Nowak U 2007 Classical Spin Models (Chichester: Wiley)

[29] Bar'yakhtar V G 1984 Zh. Eksp. Teor. Fiz. 871501

[30] Bar'yakhtar V G 1987 Fiz. Tverd. Tela 291317

[31] Baryakhtar V G and Danilevich A G 2013 Low Temp. Phys. 39993

[32] Landau L D and Lifshitz E M 1958 Statistical Physics (London: Pergamon)

[33] Garanin D A, Ishchenko V V and Panina L V 1990 Theor. Math. Phys. 82169

[34] Atxitia U, Chubykalo-Fesenko O, Chantrell R W, Nowak U and Rebei A 2009 Phys. Rev. Lett. 102057203

[35] Garanin D A 1997 Phys. Rev. B 553050

[36] Atxitia U, Chubykalo-Fesenko O, Kazantseva N, Hinzke D, Nowak U and Chantrell R W 2007 Appl. Phys. Lett. 91232507

[37] Callen H and Callen E 1966 J. Phys. Chem. Solids 271271

[38] Mryasov O N, Nowak U, Guslienko K and Chantrell R W 2005 Eur. Phys. Lett. 69805

[39] Chubykalo-Fesenko O, Nowak U, Chantrell R W and Garanin D 2006 Phys. Rev. B 74094436

[40] Chen K and Landau D P 1993 Phys. Rev. B 493266

[41] Evans R F L, Atxitia U and Chantrell R W 2015 Phys. Rev. B 91144425

[42] Garanin D A 1991 Physica A 172470

[43] Blum K 1996 Density Matrix Theory and Applications (Berlin: Springer)

[44] Nieves P, Serantes D, Atxitia U and Chubykalo-Fesenko O 2014 Phys. Rev. B 90104428

[45] Garanin D A 1992 Z. Phys. B 8677

[46] Koopmans B, Malinowski G, Dalla Longa F, Steiauf D, Fahnle M, Roth T, Cinchetti M and Aeschlimann M 2010 Nat. Mater. 9259

[47] Atxitia U, Nieves P and Chubykalo-Fesenko O 2012 Phys. Rev. B 86104414

[48] Xu L and Zhang S 2013 J. Appl. Phys. 113163911

[49] Atxitia U and Chubykalo-Fesenko O 2011 Phys. Rev. B 84144414

[50] Brown W F 1963 Phys. Rev. 1301677

[51] Brown W 1979 IEEE Trans. Magn. 151196

[52] Garanin D A and Chubykalo-Fesenko O 2004 Phys. Rev. B 70212409

[53] Evans R F L, Hinzke D, Atxitia U, Nowak U, Chantrell R W and Chubykalo-Fesenko O 2012 Phys. Rev. B 85014433

[54] Mentink J H, Tretyakov M V, Fasolino A, Katsnelson M I and Rasing T 2010 J. Phys.: Condens. Matter 22176001

[55] Ma P W and Dudarev S L 2011 Phys. Rev. B 83134418

[56] Kazantseva N, Hinzke D, Nowak U, Chantrell R W, Atxitia U and Chubykalo-Fesenko O 2008 Phys. Rev. B 77184428

[57] Kazantseva N, Hinzke D, Chantrell R W and Nowak U 2009 Eur. Phys. Lett. 8627006

[58] Gomonay E V and Loktev V M 2014 Low Temp. Phys. 4017

[59] Nieves P, Atxitia U, Chantrell R W and Chubykalo-Fesenko O 2015 Low Temp. Phys. 41739

[60] Schlickeiser F, Atxitia U, Wienholdt S, Hinzke D, ChubykaloFesenko O and Nowak U 2012 Phys. Rev. B 86214416

[61] Wangsness R K 1953 Phys. Rev. 911085

[62] Wangsness R K 1954 Phys. Rev. 9368

[63] Stanciu C D, Kimel A V, Hansteen F, Tsukamoto A, Itoh A, Kirilyuk A and Rasing T 2006 Phys. Rev. B 73220402

[64] Atxitia U, Barker J, Chantrell R W and Chubykalo-Fesenko O 2014 Phys. Rev. B 89224421

[65] Liechtenstein A I, Katsnelson M I and Gubanov V A 1984 J. Phys. F: Met. Phys. 14 L125 
[66] Liechtenstein A, Katsnelson M, Antropov V and Gubanov V 1987 J. Magn. Magn. Mater. 6765

[67] Katsnelson M I and Lichtenstein A I 2000 Phys. Rev. B 618906

[68] Katsnelson M I and Lichtenstein A I 2002 Eur. Phys. J. B 309

[69] Katsnelson M I, Kvashnin Y O, Mazurenko V V and Lichtenstein A I 2010 Phys. Rev. B 82100403

[70] Secchi A, Lichtenstein A and Katsnelson M 2015 Ann. Phys. 36061

[71] Hinzke D and Nowak U 2000 J. Magn. Magn. Mater. 221365

[72] Thiele J U, Coffey K R, Toney M F, Hedstrom J A and Kellock A J 2002 J. Appl. Phys. 916595

[73] Okamoto S, Kikuchi N, Kitakami O, Miyazaki T, Shimada Y and Fukamichi K 2002 Phys. Rev. B 6624413

[74] Hinzke D, Nowak U, Mryasov O N and Chantrell R W 2007 Appl. Phys. Lett. 90082507

[75] Hinzke D, Kazantseva N, Nowak U, Mryasov O N, Asselin P and Chantrell R W 2008 Phys. Rev. B 77094407

[76] Atxitia U, Hinzke D, Chubykalo-Fesenko O, Nowak U, Kachkachi H, Mryasov O N, Evans R F and Chantrell R W 2010 Phys. Rev. B 82134440

[77] Campana L S, D’Auria A C, D’Ambrosio M, Esposito U, De Cesare L and Kamieniarz G 1984 Phys. Rev. B 302769

[78] Hinzke D, Atxitia U, Carva K, Nieves P, ChubykaloFesenko O, Oppeneer P M and Nowak U 2015 Phys. Rev. B 92054412

[79] Mathias S et al 2012 Proc. Natl Acad. Sci. USA 1094792

[80] Soven P 1967 Phys. Rev. 156809

[81] Kazantseva N, Nowak U, Chantrell R W, Hohlfeld J and Rebei A 2008 Eur. Phys. Lett. 8127004

[82] Radu I et al 2015 Spin 051550004

[83] Beaurepaire E, Merle J C, Daunois A and Bigot J Y 1996 Phys. Rev. Lett. 764250

[84] Anisimov S I, Kapeliovich B L and Perelman T L 1974 Zh. Eksp. Teor. Fiz, 66375

[85] Atxitia U, Chubykalo-Fesenko O, Walowski J, Mann A and Münzenberg M 2010 Phys. Rev. B 81174401

[86] Illg C, Haag M and Fähnle M 2013 Phys. Rev. B 88214404

[87] Battiato M, Carva K and Oppeneer P M 2010 Phys. Rev. Lett. $\mathbf{1 0 5} 027203$

[88] Roth T, Schellekens A J, Alebrand S, Schmitt O, Steil D, Koopmans B, Cinchetti M and Aeschlimann M 2012 Phys. Rev. X 2021006

[89] Wietstruk M, Melnikov A, Stamm C, Kachel T, Pontius N, Sultan M, Gahl C, Weinelt M, Dürr H A and Bovensiepen U 2011 Phys. Rev. Lett. 106127401

[90] Koopmans B, Ruigrok J J M, Longa F D and de Jonge W J M 2005 Phys. Rev. Lett. 95267207

[91] Sultan M, Atxitia U, Melnikov A, Chubykalo-Fesenko O and Bovensiepen U 2012 Phys. Rev. B 85184407

[92] Bovensiepen U 2007 J. Phys.: Condens. Matter 19083201

[93] Frietsch B, Bowlan J, Carley R, Teichmann M, Wienholdt S, Hinzke D, Nowak U, Carva K, Oppeneer P M and Weinelt M 2015 Nat. Commun. 68262

[94] Mendil J, Nieves P, Chubykalo-Fesenko O, Walowski J, Santos T, Pisana S and Münzenberg M 2014 Sci. Rep. 43980

[95] Kimling J, Wilson R B, Hebler B, Albrecht M and Cahill D G 2014 Phys. Rev. B 90224408

[96] Barker J, Evans R F L, Chantrell R W, Hinzke D and Nowak U 2010 Appl. Phys. Lett. 97192504

[97] Ellis M O A and Chantrell R W 2015 Appl. Phys. Lett. 106162407

[98] Greaves S J, Muraoka H and Kanai Y 2015 J. Appl. Phys. 117 17C505
[99] McDaniel T W 2012 J. Appl. Phys. 112013914

[100] Kilic U, Finocchio G, Hauet T, Florez S H, Aktas G and Ozatay1 O 2012 Appl. Phys. Lett. 101252407

[101] Wang H, Sann C K, Yuan Z and Pantelis S A 2014 IEEE Trans. Magn. $\mathbf{5 0} 7100804$

[102] Vogler C, Abert C, Bruckner F and Suess D 2014 Phys. Rev. B 90214431

[103] Stanciu C D, Hansteen F, Kimel A V, Kirilyuk A, Tsukamoto A, Itoh A and Rasing T 2007 Phys. Rev. Lett. 99047601

[104] Vahaplar K, Kalashnikova A M, Kimel A V, Hinzke D, Nowak U, Chantrell R, Tsukamoto A, Itoh A, Kirilyuk A and Rasing T 2009 Phys. Rev. Lett. 103117201

[105] Vahaplar K et al 2012 Phys. Rev. B 85104402

[106] Stöhr J and Siegmann H C 2006 Magnetism vol 5 (Berlin: Springer)

[107] Atxitia U, Ostler T, Barker J, Evans R F L, Chantrell R W and Chubykalo-Fesenko O 2013 Phys. Rev. B 87224417

[108] Atxitia U, Ostler T A, Chantrell R W and ChubykaloFesenko O 2015 Appl. Phys. Lett. 107144425

[109] Suarez O J, Nieves P, Laroze D, Altbir D and Chubykalo-Fesenko O 2015 Phys. Rev. B 92144425

[110] Oniciuc E, Stoleriu L, Cimpoesu D and Stancu A 2014 Appl. Phys. Lett. 104222404

[111] Nieves P and Chubykalo-Fesenko O 2016 Phys. Rev. Appl. 5014006

[112] Parkin S S P 2003 US Patent 6834005

[113] Bulaevskiŭ L N and Ginzburg V L 1964 Sov. Phys._JETP 18530

[114] Kötzler J, Garani D A, Hartl M and Jahn L 1993 Phys. Rev. Lett. 71177

[115] Hartl-Malang M, Kötzler J and Garanin D A 1995 Phys. Rev. B 518974

[116] Yamaguchi A, Ono T, Nasu S, Miyake K, Mibu K and Shinjo T 2004 Phys. Rev. Lett. 92077205

[117] Kläui M, Vaz C A F, Bland J A C, Wernsdorfer W, Faini G, Cambril E, Heyderman L J, Nolting F and Rüdiger U 2005 Phys. Rev. Lett. 94106601

[118] Li Z and Zhang S 2004 Phys. Rev. Lett. 92207203

[119] Thiaville A, Nakatani Y, Miltat J and Suzuki N 2005 Eur. Phys. Lett. 69990

[120] Berger L 1978 J. Appl. Phys. 492156

[121] Slonczewski J C 1996 J. Magn. Magn. Mater. 159 L1

[122] Schieback C, Hinzke D, Kläui M, Nowak U and Nielaba P 2009 Phys. Rev. B 80214403

[123] Haney P M and Stiles M D 2009 Phys. Rev. B 80094418

[124] Oniciuc E, Stoleriu L and Stancu A 2014 J. Magn. Magn. Mater. 35299

[125] Ramsay A J, Roy P E, Haigh J A, Otxoa R M, Irvine A C, Janda T, Campion R P, Gallagher B L and Wunderlich J 2015 Phys. Rev. Lett. 114067202

[126] Hinzke D and Nowak U 2011 Phys. Rev. Lett. 107027205

[127] Wang X S and Wang X R 2014 Phys. Rev. B 90014414

[128] Schlickeiser F, Ritzmann U, Hinzke D and Nowak U 2014 Phys. Rev. Lett. 113097201

[129] Yan P, Cao Y and Sinova J 2015 Phys. Rev. B 92100408

[130] Stärk M et al 2015 Nanotechnology 26205302

[131] Ostler T A, Ellis M O, Hinzke D and Nowak U 2014 Phys. Rev. B 90094402

[132] Lebecki K M 2015 J. Appl. Phys. 117 17E308

[133] Lebecki K M, Hinzke D, Nowak U and Chubykalo-Fesenko O 2012 Phys. Rev. B 86094409

[134] Lebecki K M and Nowak U 2013 J. Appl. Phys. 113023906

[135] Lebecki K M and Nowak U 2014 Phys. Rev. B 89014421 